\title{
Enantioselective Synthesis of Guaianolides in the Osmitopsin Family by Domino Metathesis
}

\author{
André Barthel, Felix Kaden, Anne Jäger, and Peter Metz* \\ Fachrichtung Chemie und Lebensmittelchemie, Organische Chemie I, Technische Universität Dresden, \\ Bergstrasse 66, 01069 Dresden, Germany
}

Supporting Information 


\section{Table of contents}

1. General information

2. Synthesis of guaianolides $\mathbf{1 - 3}$

Trienyne 13

(-)-Clavukerin A (8) using Grubbs catalyst G-II

(-)-Clavukerin A (8) using Grubbs catalysts G-I/G-II

Diepoxide 15

Epoxy alcohol 16

Epoxy ketone 17 by oxidation of epoxy alcohol 16

Dienone 18

Dienol 19

Epoxy alcohol 20 and epoxy alcohol 21 by epoxidation of dienol 19

Epoxy lactone 22

Enamino lactone 24

$\alpha$-Methylene lactone 3

Epoxy lactone 5

Enamino lactone $\mathbf{S 1}$

$\alpha$-Methylene lactone $\mathbf{2}$ by DIBAL reduction of enamino lactone $\mathbf{S 1}$

$\alpha$-Methylene lactone 2 by alkylation of epoxy lactone 5 


\section{General information}

THF and $\mathrm{CH}_{2} \mathrm{Cl}_{2}$ were dried and purified by passage through a MB-SPS-800 device using molecular sieves. All other commercially available reagents were used as received. Reactions were performed under argon atmosphere. Thin layer chromatography (TLC) was performed on Merck silica gel $60 \mathrm{~F}_{254}$ $0.2 \mathrm{~mm}$ precoated plates. Product spots were visualized by UV light at $254 \mathrm{~nm}$ and subsequently developed using anisaldehyde solution as appropriate. Flash column chromatography was carried out using silica gel (Merck, particle size 40-63 microns). Melting points were measured on a Wagner \& Munz PolyTherm A and are uncorrected. Infrared spectra were recorded on a THERMONICOLET Avatar 360 instrument using ATR. NMR spectra were recorded on a Bruker AC $300 \mathrm{P}\left(300 \mathrm{MHz}{ }^{1} \mathrm{H}\right.$, $\left.75 \mathrm{MHz}{ }^{13} \mathrm{C}, 282 \mathrm{MHz}{ }^{19} \mathrm{~F}\right)$, Bruker DRX $500 \mathrm{P}\left(500 \mathrm{MHz}{ }^{1} \mathrm{H}, 125 \mathrm{MHz}{ }^{13} \mathrm{C}\right)$, or on a Bruker Avance III $600\left(600 \mathrm{MHz}{ }^{1} \mathrm{H}, 150 \mathrm{MHz}{ }^{13} \mathrm{C}\right)$ spectrometer. Chemical shifts $(\delta)$ are quoted in parts per million (ppm) downfield of tetramethylsilane, using residual proton-containing solvent as internal standard $\left(\mathrm{CDCl}_{3}\right.$ at $\left.7.27 \mathrm{ppm}\right)$. Abbreviations used in the description of resonances are: $\mathrm{s}$ (singlet), $\mathrm{d}$ (doublet), $\mathrm{t}$ (triplet), q (quartet), br (broad). Coupling constants $(J)$ are quoted to the nearest $0.1 \mathrm{~Hz}$. Despite our best efforts to remove all solvent residues from the synthesized samples, some NMR spectra still contain solvent signals. Mass spectra were recorded with an Agilent 5973N detector coupled with an Agilent 6890N GC (GC-MS, $70 \mathrm{eV}$ ) or else with a Bruker Esquire-LC (direct injection as a methanolic $\mathrm{NH}_{4} \mathrm{OAc}$ solution, ESI). HRMS spectra were recorded on a Finnigan MAT 95 (EI, $70 \mathrm{eV}$ ) or a Bruker Daltonic "Impact II" (ESI-TOF). Optical rotations were measured on a Perkin Elmer 341 LC polarimeter. Elemental analysis was performed on a Hekatech EA 3000.

\section{$\underline{\text { 2. Synthesis of guaianolides 1-3 }}$}

\section{$\underline{\text { Trienyne } 13}$}

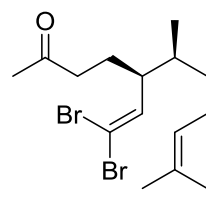

10

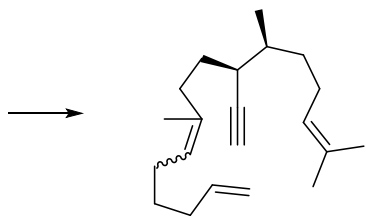

13

Hex-5-en-1-yltriphenylphosphonium bromide $(7.57 \mathrm{~g}, 17.8 \mathrm{mmol})$ in THF $(163 \mathrm{~mL})$ at $-78{ }^{\circ} \mathrm{C}$ was deprotonated with BuLi (7.65 mL, $17.6 \mathrm{mmol}, 2.3 \mathrm{M}$ in hexane). The resulting cloudy red-orange solution was stirred for $15 \mathrm{~min}$ at this temperature and then was warmed to room temperature until a clear red solution arose. Dibromoolefin 10 (1.65 g, $4.34 \mathrm{mmol})$ dissolved in THF (7 mL) was added. The reaction mixture turned immediately into cloudy red-orange solution and was stirred for $3 \mathrm{~d}$ at room temperature. After complete conversion of $\mathbf{1 0}$, the mixture was cooled to $-78{ }^{\circ} \mathrm{C}$, and $\mathrm{BuLi}(4.15$ $\mathrm{mL}, 9.56 \mathrm{mmol}, 2.3 \mathrm{M}$ in hexane) was added. After additional $90 \mathrm{~min}$, the mixture was filtered over a 
pad of silica (elution with pentane). After flash chromatography (pentane), the trienyne $\mathbf{1 3}$ was obtained as a colorless oil (inseparable mixture of $E / Z$ isomers; $d r=1: 1.8,751.6 \mathrm{mg}, 61 \%$ ).

$\mathrm{R}_{\mathrm{f}}=0.34$ (pentane); $[\alpha]_{\mathrm{D}}{ }^{25}+50.4\left(c 1.69, \mathrm{CHCl}_{3}\right) ;{ }^{1} \mathrm{H} \mathrm{NMR}\left(300 \mathrm{MHz}, \mathrm{CDCl}_{3}\right): \delta 0.95(\mathrm{~d}, J=6.4 \mathrm{~Hz}$, $3 \mathrm{H}), 1.23-1.36(\mathrm{~m}, 1 \mathrm{H}), 1.37-1.56(\mathrm{~m}, 6 \mathrm{H}), 1.57-1.64(\mathrm{~m}, 6 \mathrm{H}), 1.69(\mathrm{~s}, 3 \mathrm{H}), 1.93-2.12(\mathrm{~m}, 8 \mathrm{H})$, 2.13-2.22 (m, 1H), 2.29-2.41 (m, 1H), 4.86-5.24 (m, 4H), 5.73-5.93 (m, 1H); ${ }^{13} \mathrm{C}$ NMR $(75 \mathrm{MHz}$, $\left.\mathrm{CDCl}_{3}\right): \delta 15.26\left(\mathrm{CH}_{3}\right), 15.98\left(\mathrm{CH}_{3}\right), 17.65\left(\mathrm{CH}_{3}\right), 23.39\left(\mathrm{CH}_{3}\right), 25.64\left(\mathrm{CH}_{2}\right), 25.71\left(\mathrm{CH}_{3}\right), 27.28$ $\left(\mathrm{CH}_{2}\right), 27.37\left(\mathrm{CH}_{2}\right), 29.06\left(\mathrm{CH}_{2}\right), 29.33\left(\mathrm{CH}_{2}\right), 29.96\left(\mathrm{CH}_{2}\right), 31.32\left(\mathrm{CH}_{2}\right), 31.43\left(\mathrm{CH}_{2}\right), 33.37\left(\mathrm{CH}_{2}\right)$, $33.45\left(\mathrm{CH}_{2}\right), 35.42\left(\mathrm{CH}_{2}\right), 35.46\left(\mathrm{CH}_{2}\right), 35.60(\mathrm{CH}), 36.04(\mathrm{CH}), 36.45(\mathrm{CH}), 70.13(\mathrm{CH}), 70.23(\mathrm{CH})$, $85.91(\mathrm{C}), 85.94(\mathrm{C}), 114.29\left(\mathrm{CH}_{2}\right), 114.31\left(\mathrm{CH}_{2}\right), 124.52(\mathrm{CH}), 124.55(\mathrm{CH}), 124.81(\mathrm{CH}), 125.66$ (CH), 131.36 (C), 131.38 (C), 134.68 (C), 134.90 (C), 138.99 (CH); IR (ATR): v = 3308, 3074, 2962, 2923, 2856, 2110, 1640, 1486, 1473, 1447, 1378, 1239, 1115, 991, 910, 828, $630 \mathrm{~cm}^{-1}$; GC-MS (EI): m/z = 286 (1), 271 (4), 243 (4), 147 (28), 135 (25), 133 (29), 121 (38), 119 (38), 109 (29), 81 (70), 79 (44), 69 (92), 67 (54), 55 (64), 53 (22), 41 (100), 39 (17); HRMS (EI): m/z calcd for $\mathrm{C}_{21} \mathrm{H}_{34}[\mathrm{M}]^{+}$: 286.2661; found: 286.2665 .

\section{$\underline{(-)-C l a v u k e r i n ~ A ~(8) ~ u s i n g ~ G r u b b s ~ c a t a l y s t ~ G-I I ~}$}

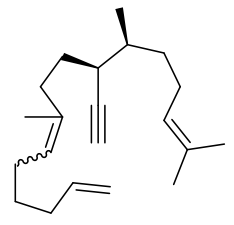

13

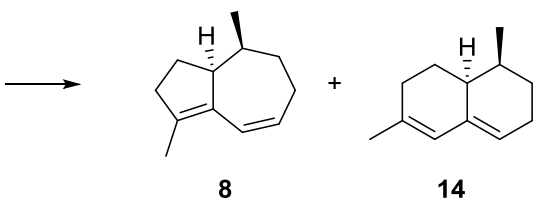

$(13$

1)

Second generation Grubbs catalyst G-II $(93.3 \mathrm{mg}, 110 \mu \mathrm{mol})$ and $p$-benzoquinone $(23.7 \mathrm{mg}$, $219 \mu \mathrm{mol})$ were placed in a round bottom flask and dissolved in $\mathrm{CH}_{2} \mathrm{Cl}_{2}(74 \mathrm{~mL})$. A solution of trienyne 13 (628 mg, $2.19 \mathrm{mmol})$ in $\mathrm{CH}_{2} \mathrm{Cl}_{2}(4 \mathrm{~mL})$ was added, and the mixture was stirred for $2 \mathrm{~h}$ at reflux. Then further catalyst G-II $(46.7 \mathrm{mg}, 54.9 \mu \mathrm{mol})$ in $\mathrm{CH}_{2} \mathrm{Cl}_{2}(3 \mathrm{~mL})$ was added, and stirring was continued for $2 \mathrm{~h}$. After complete conversion of $\mathbf{1 3}$ was indicated by GC, the dark blue mixture was filtered over a pad of silica (elution with pentane). Purification by column chromatography over silica gel impregnated with silver nitrate ${ }^{1}$ (pentane/diethyl ether, $15 / 1 \rightarrow 10 / 1$ ) provided pure $(-$ )-clavukerin $\mathrm{A}^{2}(\mathbf{8}, 328 \mathrm{mg}, 92 \%)$ as a colorless oil. 


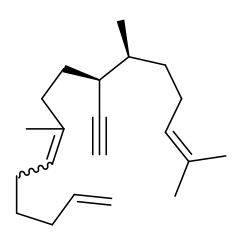

13

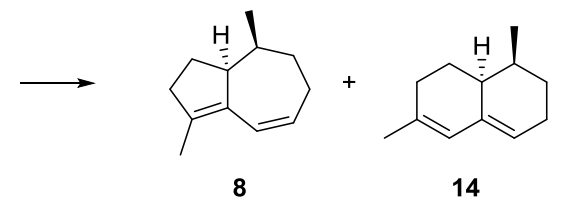

(25 : 1)

First generation Grubbs catalyst G-I $(20.5 \mathrm{mg}, 25.0 \mu \mathrm{mol})$ was placed in a round bottom flask and dissolved in $\mathrm{CH}_{2} \mathrm{Cl}_{2}(17 \mathrm{~mL})$. A solution of trienyne $13(143.0 \mathrm{mg}, 499.1 \mu \mathrm{mol})$ in $\mathrm{CH}_{2} \mathrm{Cl}_{2}(2 \mathrm{~mL})$ was added, and the mixture was stirred at reflux. After $2 \mathrm{~h}$ and after $5 \mathrm{~h}$ in total, further catalyst G-I (10.3 $\mathrm{mg}, 12.5 \mu \mathrm{mol})$ in $\mathrm{CH}_{2} \mathrm{Cl}_{2}(2 \mathrm{~mL})$ was added. Then catalyst G-II $(21.3 \mathrm{mg}, 25 \mu \mathrm{mol})$ was added after a total reaction time of $7 \mathrm{~h}$. Stirring was continued for $2 \mathrm{~h}$ at reflux, further catalyst G-II (10.6 mg, $12.5 \mu \mathrm{mol})$ in $\mathrm{CH}_{2} \mathrm{Cl}_{2}(2 \mathrm{~mL})$ was added, and stirring was continued for another $2 \mathrm{~h}$. After complete conversion of $\mathbf{1 3}$ was indicated by GC, the dark blue mixture was filtered over a pad of silica (elution with pentane). Purification by column chromatography over silica gel impregnated with silver nitrate ${ }^{1}$ (pentane/diethyl ether, $15 / 1 \rightarrow 10 / 1)$ provided pure $(-)$-clavukerin $\mathrm{A}^{2}(\mathbf{8}, 77.7 \mathrm{mg}, 96 \%)$ as a colorless oil.

\section{$\underline{\text { Diepoxide } 15}$}

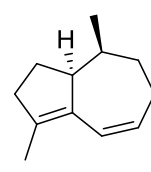

8

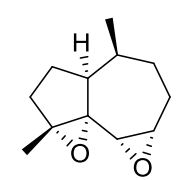

15

(-)-Clavukerin A (8) $(85.2 \mathrm{mg}, 525 \mu \mathrm{mol})$ was dissolved in dimethoxymethane $(15 \mathrm{~mL})$ and acetonitrile $(5.3 \mathrm{~mL})$ at $0{ }^{\circ} \mathrm{C} .1,1,1$-Trifluoroacetone $(588 \mathrm{mg}, 5.25 \mathrm{mmol})$ was added dropwise with a precooled syringe followed by aqueous $\mathrm{Na}_{2}$ EDTA-solution $\left(11 \mathrm{~mL}, 4 \times 10^{-4} \mathrm{M}\right)$. Under vigorous stirring, a mixture of oxone $(1.61 \mathrm{~g}, 5.24 \mathrm{mmol})$ and $\mathrm{NaHCO}_{3}(684 \mathrm{mg}, 8.14 \mathrm{mmol})$ was added portionwise. The mixture should have $\mathrm{pH} 7-8$. After $1 \mathrm{~h}$, water was added until oxone and $\mathrm{NaHCO}_{3}$ were dissolved. The aqueous layer was extracted three times with $\mathrm{CH}_{2} \mathrm{Cl}_{2}$. After drying over magnesium sulfate, purification by flash chromatography (pentane/diethyl ether, 4/1) furnished diepoxide 15 as colorless crystals (100.9 mg, 99\%).

$\mathrm{R}_{\mathrm{f}}=0.20$ (pentane/diethyl ether, 5/1); mp 90-91 ${ }^{\circ} \mathrm{C} ;[\alpha]_{\mathrm{D}}{ }^{23}+6.5\left(c 1.17, \mathrm{CH}_{2} \mathrm{Cl}_{2}\right) ;{ }^{1} \mathrm{H}$ NMR $(600 \mathrm{MHz}$, $\left.\mathrm{CDCl}_{3}\right): \delta 0.77(\mathrm{~d}, J=7.2 \mathrm{~Hz}, 3 \mathrm{H}), 1.20-1.24(\mathrm{~m}, 1 \mathrm{H}), 1.48(\mathrm{~s}, 3 \mathrm{H}), 1.49-1.52(\mathrm{~m}, 1 \mathrm{H}), 1.66-1.75(\mathrm{~m}$, $3 \mathrm{H}), 1.77-1.81(\mathrm{~m}, 1 \mathrm{H}), 1.87-1.92(\mathrm{~m}, 1 \mathrm{H}), 2.06(\mathrm{~m}, 1 \mathrm{H}), 2.23(\mathrm{dtd}, J=16 \mathrm{~Hz}, 4 \mathrm{~Hz}, 4 \mathrm{~Hz}, 1 \mathrm{H}), 2.36$ 
$(\mathrm{dd}, J=8.3 \mathrm{~Hz}, 3.4 \mathrm{~Hz}, 1 \mathrm{H}), 2.81(\mathrm{~d}, J=4.5 \mathrm{~Hz}, 1 \mathrm{H}), 3.20(\mathrm{dd}, J=4.5 \mathrm{~Hz}, 4 \mathrm{~Hz}, 1 \mathrm{H}) ;{ }^{13} \mathrm{C} \mathrm{NMR}(150$ $\left.\mathrm{MHz}, \mathrm{CDCl}_{3}\right): \delta 12.40\left(\mathrm{CH}_{3}\right), 15.50\left(\mathrm{CH}_{3}\right), 22.61\left(\mathrm{CH}_{2}\right), 26.72\left(\mathrm{CH}_{2}\right), 28.78\left(\mathrm{CH}_{2}\right), 33.11\left(\mathrm{CH}_{2}\right)$, $34.01(\mathrm{CH}), 45.92(\mathrm{CH}), 55.86(\mathrm{CH}), 58.53(\mathrm{CH}), 69.53(\mathrm{C}), 69.75(\mathrm{C})$; IR (ATR): $v$ = 3023, 2959, 2925, 2881, 1737, 1486, 1469, 1457, 1439, 1380, 1307, 1284, 1216, 1176, 1150, 1105, 1059, 1017, 962, 944, 922, 889, 859, 837, 775, 743, 697, 659, $637 \mathrm{~cm}^{-1}$. GC-MS (EI): m/z = 193 (2), 179 (4), 165 (6), 151 (9), 137 (20), 123 (15), 109 (25), 95 (28), 94 (11), 93 (21), 91 (20), 81 (25), 79 (25), 55 (22), 43 (100), 41 (35), 39 (26); anal. calcd for $\mathrm{C}_{12} \mathrm{H}_{18} \mathrm{O}_{2}$ : C, 74.19; H, 9.34; found: C, 74.16; H, 9.59.

\section{Epoxy alcohol 16}

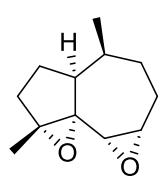

15

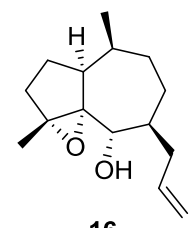

16

To a solution of $15(190.3 \mathrm{mg}, 979.6 \mu \mathrm{mol})$ in $\mathrm{THF}(5.4 \mathrm{~mL})$ at $0{ }^{\circ} \mathrm{C}$ was added $\mathrm{CuCN}(8.5 \mathrm{mg}, 94.9$ $\mu \mathrm{mol})$. Then a solution of allylmagnesium bromide in diethyl ether $(12.4 \mathrm{~mL}, 7.81 \mathrm{mmol}, 0.63 \mathrm{M})$ was added dropwise. After warming to room temperature and stirring for $3 \mathrm{~h}$ at this temperature, the solution was cooled to $0{ }^{\circ} \mathrm{C}$ again, and saturated aqueous $\mathrm{NH}_{4} \mathrm{Cl}(10 \mathrm{~mL})$ was carefully added dropwise followed by water $(25 \mathrm{~mL})$. The aqueous layer was extracted three times with diethyl ether. After drying over magnesium sulfate, column chromatography (pentane/ethyl acetate, 6/1) gave bishomoallylic alcohol 16 as a colorless oil (184.9 mg, 80\%).

$\mathrm{R}_{\mathrm{f}}=0.45$ (pentane/ethyl acetate 6/1); $[\alpha]_{\mathrm{D}}{ }^{23}+47.3\left(\right.$ c 1.49, $\left.\mathrm{CHCl}_{3}\right) ;{ }^{1} \mathrm{H}$ NMR $\left(600 \mathrm{MHz}, \mathrm{CDCl}_{3}\right): \delta$ $0.83(\mathrm{~d}, J=7.2 \mathrm{~Hz}, 3 \mathrm{H}), 1.18-1.24(\mathrm{~m}, 1 \mathrm{H}), 1.32-1.38(\mathrm{~m}, 1 \mathrm{H}), 1.44(\mathrm{~s}, 3 \mathrm{H}), 1.51-1.57(\mathrm{~m}, 2 \mathrm{H})$, 1.67-1.78 (m, 4H), 1.90-1.97 (m, 1H), 2.00-2.09 (m, 2H), 2.47-2.55 (m, 2H), $2.80(\mathrm{~d}, J=4.5 \mathrm{~Hz}, 1 \mathrm{H})$, $3.42(\mathrm{dd}, J=9.4 \mathrm{~Hz}, 4.5 \mathrm{~Hz}, 1 \mathrm{H}), 5.01-5.09$ (m, 2H), 5.84 (dddd, $J=16.8 \mathrm{~Hz}, 10.4 \mathrm{~Hz}, 7.8 \mathrm{~Hz}, 6.3 \mathrm{~Hz}$, $1 \mathrm{H}) ;{ }^{13} \mathrm{C}$ NMR $\left(150 \mathrm{MHz}, \mathrm{CDCl}_{3}\right): \delta 14.75\left(\mathrm{CH}_{3}\right), 16.03\left(\mathrm{CH}_{3}\right), 26.96\left(\mathrm{CH}_{2}\right), 27.19\left(\mathrm{CH}_{2}\right), 33.19$ $\left(\mathrm{CH}_{2}\right), 34.85\left(\mathrm{CH}_{2}\right), 35.21(\mathrm{CH}), 38.29\left(\mathrm{CH}_{2}\right), 43.02(\mathrm{CH}), 46.47(\mathrm{CH}), 71.32(\mathrm{C}), 72.27(\mathrm{CH}), 74.36$ (C), $116.32\left(\mathrm{CH}_{2}\right), 137.24(\mathrm{CH})$; IR (ATR): $v=3461,3074,2953,2921,2853,2030,1716,1639$, 1486, 1446, 1414, 1396, 1379, 1334, 1296, 1256, 1165, 1047, 1019, 995, 908, 885, 856, 813, 785, 739, 699, 673, $624 \mathrm{~cm}^{-1}$; ESI-MS: $\mathrm{m} / \mathrm{z}=259[\mathrm{M}+\mathrm{Na}]^{+}, 237[\mathrm{M}+\mathrm{H}]^{+}$; anal. calcd for $\mathrm{C}_{15} \mathrm{H}_{24} \mathrm{O}_{2}: \mathrm{C}, 76.23 ; \mathrm{H}$, 10.24; found: C, $76.32 ; \mathrm{H}, 10.21$. 
Epoxy ketone 17 by oxidation of epoxy alcohol 16

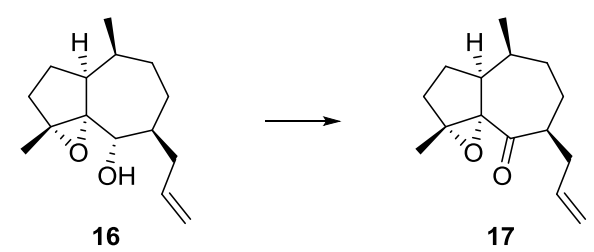

A solution of alcohol $16(356.8 \mathrm{mg}, 1.51 \mathrm{mmol})$ in DMF $(2 \mathrm{~mL})$ was added to PDC $(1.74 \mathrm{~g}, 4.63$ mmol) in DMF $(4.5 \mathrm{~mL})$ at room temperature. The resulting dark orange solution was stirred overnight. After complete conversion of 16, the mixture was diluted with water $(5 \mathrm{~mL})$. After extraction for three times with diethyl ether, column chromatography (pentane/ethyl acetate, 10/1) yielded ketone 17 as a colorless, crystalline substance (337.4 mg, 95\%).

$\mathrm{R}_{\mathrm{f}}=0.35$ (pentane/ethyl acetate, 10/1); mp $61.2{ }^{\circ} \mathrm{C} ;[\alpha]_{\mathrm{D}}{ }^{25}-19.0\left(c 1.46, \mathrm{CHCl}_{3}\right) ;{ }^{1} \mathrm{H}$ NMR $(600 \mathrm{MHz}$, $\left.\mathrm{CDCl}_{3}\right): \delta 0.89(\mathrm{~d}, J=7.2 \mathrm{~Hz}, 3 \mathrm{H}), 1.45-1.51(\mathrm{~m}, 1 \mathrm{H}), 1.53(\mathrm{~s}, 3 \mathrm{H}), 1.57-1.64(\mathrm{~m}, 1 \mathrm{H}), 1.66-1.76(\mathrm{~m}$, 2H), 1.76-1.80 (m, 1H), 1.80-1.85 (m, 1H), 1.89 (ddd, $J=14.5 \mathrm{~Hz}, 10.3 \mathrm{~Hz}, 7.5 \mathrm{~Hz}, 1 \mathrm{H}), 1.95-2.04$ $(\mathrm{m}, 2 \mathrm{H}), 2.08-2.16(\mathrm{~m}, 1 \mathrm{H}), 2.49-2.64(\mathrm{~m}, 3 \mathrm{H}), 5.01-5.07(\mathrm{~m}, 2 \mathrm{H}), 5.69-5.78(\mathrm{~m}, 1 \mathrm{H}) ;{ }^{13} \mathrm{C} \mathrm{NMR}$ $\left(150 \mathrm{MHz}, \mathrm{CDCl}_{3}\right): \delta 16.37\left(\mathrm{CH}_{3}\right), 16.84\left(\mathrm{CH}_{3}\right), 25.39\left(\mathrm{CH}_{2}\right), 25.87\left(\mathrm{CH}_{2}\right), 33.01\left(\mathrm{CH}_{2}\right), 33.24\left(\mathrm{CH}_{2}\right)$, $33.73\left(\mathrm{CH}_{2}\right), 35.84\left(\mathrm{CH}_{2}\right), 46.12(\mathrm{CH}), 50.21(\mathrm{CH}), 74.87(2 \mathrm{C}, \mathrm{C}), 116.97\left(\mathrm{CH}_{2}\right), 136.15(\mathrm{CH})$, 209.53 (C); IR (ATR): v = 3081, 2958, 2932, 2881, 2856, 2138, 2056, 2030, 2009, 1917, 1843, 1734, $1702,1640,1463,1444,1394,1373,1324,1259,1181,1160,1114,1054,1002,951,919,892,858$, 813, 720, 660, $621 \mathrm{~cm}^{-1}$; GC-MS (EI): m/z = 234 (3), 219 (4), 206 (4), 205 (11), 191 (30), 177 (24), 149 (19), 135 (30), 123 (57), 107 (37), 95 (36), 69 (26), 67 (43), 55 (62), 53 (41), 43 (100), 41 (99), 40 (11), 39 (53); anal. calcd for $\mathrm{C}_{15} \mathrm{H}_{22} \mathrm{O}_{2}$ : C, 76.88; H, 9.46; found: C, 76.98; H, 9.63.

\section{$\underline{\text { Dienone } 18}$}
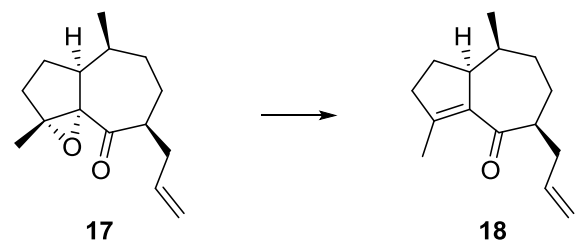

To a solution of epoxy ketone $17(42.3 \mathrm{mg}, 180.5 \mu \mathrm{mol})$ in 1,2-dimethoxyethane ( $2 \mathrm{~mL})$ was added $\operatorname{Mo}(\mathrm{CO})_{6}(47.8 \mathrm{mg}, 181 \mu \mathrm{mol})$. After stirring the mixture under reflux for $5 \mathrm{~h}$, it was filtered over a pad of silica (elution with diethyl ether). Chromatographic purification (pentane/ethyl acetate, 30/1) yielded the unsaturated ketone $\mathbf{1 8}$ (38.5 mg, $176.3 \mu \mathrm{mol}, 98 \%)$ as a colorless oil. 
$\mathrm{R}_{\mathrm{f}}=0.32$ (pentane/ethyl acetate, 30/1); $[\alpha]_{\mathrm{D}}{ }^{23}+148.1\left(\mathrm{c} 1.38, \mathrm{CHCl}_{3}\right) ;{ }^{1} \mathrm{H} \mathrm{NMR}\left(300 \mathrm{MHz}, \mathrm{CDCl}_{3}\right): \delta$ $0.72(\mathrm{~d}, J=6.7 \mathrm{~Hz}, 3 \mathrm{H}), 1.35-1.67(\mathrm{~m}, 4 \mathrm{H}), 1.75-1.84(\mathrm{~m}, 2 \mathrm{H}), 1.91-2.05(\mathrm{~m}, 2 \mathrm{H}), 2.08$ (br s, 3H), 2.30-2.63 (m, 4H), 3.22-3.34 (m, 1H), 4.95-5.09 (m, 2H), 5.80 (dddd, $J=17.1 \mathrm{~Hz}, 10.3 \mathrm{~Hz}, 8.0 \mathrm{~Hz}$, $5.5 \mathrm{~Hz}, 1 \mathrm{H}) ;{ }^{13} \mathrm{C}$ NMR $\left(75 \mathrm{MHz}, \mathrm{CDCl}_{3}\right): \delta 12.06\left(\mathrm{CH}_{3}\right), 16.70\left(\mathrm{CH}_{3}\right), 26.03\left(\mathrm{CH}_{2}\right), 27.57\left(\mathrm{CH}_{2}\right)$, $35.39\left(\mathrm{CH}_{2}\right), 35.95(\mathrm{CH}), 37.12\left(\mathrm{CH}_{2}\right), 39.43\left(\mathrm{CH}_{2}\right), 50.24(\mathrm{CH}), 52.04(\mathrm{CH}), 115.95\left(\mathrm{CH}_{2}\right), 136.42$ (C), 137.34 (CH), 155.53 (C), 203.45 (C); IR (ATR): v = 3074, 2955, 2912, 2869, 1844, 1773, 1735, 1718, 1697, 1671, 1654, 1609, 1507, 1473, 1457, 1433, 1396, 1374, 1290, 1244, 1185, 1106, 1043, 909, 863, 762, $639 \mathrm{~cm}^{-1}$; GC-MS (EI): m/z = 218 (66), 203 (17), 177 (13), 175 (21), 161 (32), 149 (28), 109 (38), 108 (25), 107 (45), 105 (24), 93 (37), 91 (49), 81 (43), 80 (30), 79 (100), 77 (60), 65 (21), 55 (32), 41 (58), 39 (39); anal. calcd for $\mathrm{C}_{15} \mathrm{H}_{22} \mathrm{O}: \mathrm{C}, 82.52$; H, 10.16; found: $\mathrm{C}, 82.56$; $\mathrm{H}, 10.39$.

\section{$\underline{\text { Dienol } 19}$}

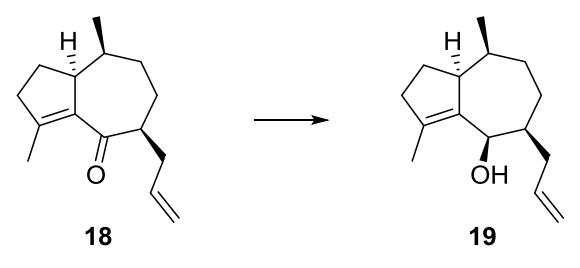

To a solution of unsaturated ketone $\mathbf{1 8}(127.7 \mathrm{mg}, 584.9 \mu \mathrm{mol})$ in THF $(25.5 \mathrm{~mL})$ cooled to $-78{ }^{\circ} \mathrm{C}$ was added L-selectride $(1.75 \mathrm{~mL}, 1.75 \mathrm{mmol}, 1 \mathrm{M}$ in THF), and then the mixture was warmed to room temperature for $60 \mathrm{~min}$. After addition of $\mathrm{NaOH}\left(3.5 \mathrm{~mL}, 2 \mathrm{M}\right.$ in water) and $\mathrm{H}_{2} \mathrm{O}_{2}(2.2 \mathrm{~mL}, 30 \mathrm{wt} \%)$ at $0{ }^{\circ} \mathrm{C}$, stirring was continued for additional $30 \mathrm{~min}$. The aqueous layer was diluted with water and extracted with $20 \mathrm{~mL} \mathrm{CH}_{2} \mathrm{Cl}_{2}$. The combined organic layers were dried over magnesium sulfate. Flash chromatography (pentane/ethyl acetate, 10/1) gave allylic alcohol 19 (126.9 mg, 99\%) as a colorless oil.

$\mathrm{R}_{\mathrm{f}}=0.52$ (pentane/ethyl acetate, 10/1); $[\alpha]_{\mathrm{D}}{ }^{21}+93.9\left(c 1.06, \mathrm{CHCl}_{3}\right) ;{ }^{1} \mathrm{H} \mathrm{NMR}\left(600 \mathrm{MHz}, \mathrm{CDCl}_{3}\right): \delta$ $0.85(\mathrm{~d}, J=7.2 \mathrm{~Hz}, 3 \mathrm{H}), 1.18(\mathrm{~d}, J=3.0 \mathrm{~Hz}, 1 \mathrm{H}), 1.27-1.32(\mathrm{~m}, 1 \mathrm{H}), 1.41-1.60(\mathrm{~m}, 3 \mathrm{H}), 1.64-1.70$ (m, 1H), 1.78 (s, 3H), $1.82(\mathrm{ddq}, J=10.4 \mathrm{~Hz}, 7.0 \mathrm{~Hz}, 3.6 \mathrm{~Hz}, 1 \mathrm{H}), 1.86-1.99$ (m, 2H), 2.10 (ddd, $J=$ 14.1 Hz, 7.7 Hz, 7.6 Hz, 1H), 2.17-2.36 (m, 3H), 2.90-2.97 (m, 1H), 4.48 (br s, 1H), 5.00-5.10 (m, 2H), $5.84(\mathrm{ddt}, J=17.1 \mathrm{~Hz}, 10.1 \mathrm{~Hz}, 7.1 \mathrm{~Hz}, 1 \mathrm{H}) ;{ }^{13} \mathrm{C} \mathrm{NMR}\left(150 \mathrm{MHz}, \mathrm{CDCl}_{3}\right): \delta 13.06\left(\mathrm{CH}_{3}\right), 14.99$ $\left(\mathrm{CH}_{3}\right), 23.41\left(\mathrm{CH}_{2}\right), 28.04\left(\mathrm{CH}_{2}\right), 36.28(\mathrm{CH}), 36.54\left(\mathrm{CH}_{2}\right), 37.58\left(\mathrm{CH}_{2}\right), 39.66\left(\mathrm{CH}_{2}\right), 43.34(\mathrm{CH})$, $53.59(\mathrm{CH}), 69.74(\mathrm{CH}), 115.97\left(\mathrm{CH}_{2}\right), 138.10(\mathrm{CH}), 139.01(\mathrm{C}), 140.49(\mathrm{C})$; IR (ATR): $v=3619$, $34913074,2914,2866,2029,1640,1473,1445,1380,1326,1253,1154,1093,1039$, 993, 971, 909, 842, $641 \mathrm{~cm}^{-1}$; GC-MS (EI): m/z = 220 (36), 205 (20), 202 (6), 193 (4), 187 (9), 179 (30), 178 (43), 177 (24), 163 (20), 161 (39), 149 (15), 145 (18), 138 (15), 137 (46), 135 (23), 131 (14), 123 (20), 121 (28), 119 (24), 117 (14), 111 (71), 110 (23), 109 (85), 108 (16), 107 (33), 105 (43), 97 (11), 95 (59), 
94 (13), 93 (60), 91 (54), 81 (100), 79 (59), 77 (41), 69 (20), 67 (50), 65 (18), 55 (60), 53 (28), 43 (26), 41 (69), 39 (27); anal. calcd for $\mathrm{C}_{15} \mathrm{H}_{24} \mathrm{O}: \mathrm{C}, 81.76 ; \mathrm{H}, 10.98$; found: $\mathrm{C}, 81.41 ; \mathrm{H}, 10.90$.

Epoxy alcohol 20 and epoxy alcohol 21 by epoxidation of dienol 19

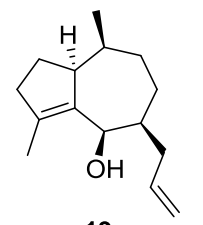

19

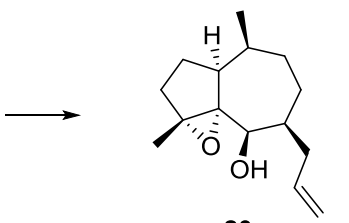

20

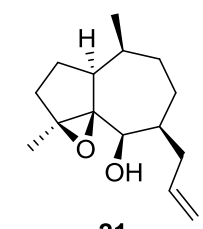

21

A solution of allyl alcohol $19(183.9 \mathrm{mg}, 834.5 \mu \mathrm{mol})$ in benzene $(10 \mathrm{~mL})$ was treated at room temperature with a solution of $\mathrm{VO}(\mathrm{acac})_{2}(10.5 \mathrm{~mL}, 21 \mu \mathrm{mol}, 0.002 \mathrm{M}$ in benzene $)$ and a solution of tert-butylhydroperoxide ( $463.4 \mu \mathrm{l}, 2.32 \mathrm{mmol}, 5 \mathrm{M}$ in benzene). The resultant mixture was stirred for $60 \mathrm{~min}$, and then saturated aqueous sodium thiosulfate solution and water were added. The aqueous layer was extracted three times with ethyl acetate. After drying over magnesium sulfate, flash chromatography (pentane/ethyl acetate, 20/1) provided alcohol $\mathbf{2 0}$ (96.3 mg, 49\%) as a white solid and alcohol 21 (83.0 $\mathrm{mg}, 42 \%)$ as a colorless oil.

20: $\mathrm{R}_{\mathrm{f}}=0.13$ (pentane/ethyl acetate, 20/1); mp $70.4{ }^{\circ} \mathrm{C} ;[\alpha]_{\mathrm{D}}{ }^{22}-3.7\left(c 0.99, \mathrm{CHCl}_{3}\right) ;{ }^{1} \mathrm{H}^{\mathrm{N} M R}(600$ $\left.\mathrm{MHz}, \mathrm{CDCl}_{3}\right): \delta 1.02(\mathrm{~d}, J=7.2 \mathrm{~Hz}, 3 \mathrm{H}), 1.21-1.31(\mathrm{~m}, 1 \mathrm{H}), 1.36(\mathrm{dt}, J=13.0 \mathrm{~Hz}, 4.0 \mathrm{~Hz}, 1 \mathrm{H}), 1.54$ (s, 3H), 1.57 (br s, 1H), 1.59-1.63 (m, 1H), 1.64-1.77 (m, 2H), 1.77-1.87 (m, 3H), 1.88-1.99 (m, 2H), $2.07(\mathrm{dt}, J=13.8 \mathrm{~Hz}, J=6.8 \mathrm{~Hz}, 1 \mathrm{H}), 2.20(\mathrm{~m}, 1 \mathrm{H}), 2.45(\mathrm{dt}, J=9.4 \mathrm{~Hz}, 2.5 \mathrm{~Hz}, 1 \mathrm{H}), 3.93(\mathrm{~s}, 1 \mathrm{H})$, 4.99-5.10 (m, 2H), $5.83(\mathrm{ddt}, J=17.1 \mathrm{~Hz}, 10.1 \mathrm{~Hz}, 7.1 \mathrm{~Hz}, 1 \mathrm{H}) ;{ }^{13} \mathrm{C}$ NMR $\left(150 \mathrm{MHz}, \mathrm{CDCl}_{3}\right): \delta$ $13.75\left(\mathrm{CH}_{3}\right), 17.91\left(\mathrm{CH}_{3}\right), 23.56\left(\mathrm{CH}_{2}\right), 28.14\left(\mathrm{CH}_{2}\right), 33.41\left(\mathrm{CH}_{2}\right), 36.16\left(\mathrm{CH}_{2}\right), 36.88(\mathrm{CH}), 39.80$ $\left(\mathrm{CH}_{2}\right), 41.57(\mathrm{CH}), 49.14(\mathrm{CH}), 71.82(\mathrm{C}), 74.28(\mathrm{C}), 75.98(\mathrm{CH}), 116.49\left(\mathrm{CH}_{2}\right), 137.54(\mathrm{CH})$; IR (ATR): $v=3575,3074,2954,2925,2899,2865,1733,1641,1473,1449,1413,1381,1322,1293$, 1237, 1200, 1167, 1092, 1041, 1021, 990, 964, 938, 910, 892, 855, 830, 776, 737, 712, 661, 636, 606 $\mathrm{cm}^{-1}$; GC-MS (EI): m/z = 235 (2), 221 (2), 218 (2), 205 (10), 193 (15), 178 (11), 175 (22), 163 (10), 137 (22), 135 (16), 133 (11), 125 (15), 123 (17), 121 (13), 119 (19), 111 (13), 109 (27), 107 (21), 105 (18), 97 (19), 96 (11), 95 (35), 93 (24), 91 (27), 83 (20), 81 (41), 79 (31), 77 (19), 71 (11), 69 (21), 67 (31), 57 (10), 55 (46), 53 (20), 43 (100), 41 (66), 39 (29); anal. calcd for $\mathrm{C}_{15} \mathrm{H}_{24} \mathrm{O}_{2}$ : C, 76.23; H, 10.24; found: C, 75.96; H, 10.38 .

21: $\mathrm{R}_{\mathrm{f}}=0.35$ (pentane/ethyl acetate, 20/1); $[\alpha]_{\mathrm{D}}{ }^{23}+47.2\left(c 0.53, \mathrm{CHCl}_{3}\right) ;{ }^{1} \mathrm{H}$ NMR $\left(600 \mathrm{MHz}, \mathrm{CDCl}_{3}\right)$ : $\delta 0.99(\mathrm{~d}, J=7.2 \mathrm{~Hz}, 3 \mathrm{H}), 1.25-1.30(\mathrm{~m}, 2 \mathrm{H}), 1.35$ (s, 3H), $1.41(\mathrm{dt}, J=12.4 \mathrm{~Hz}, 8.1 \mathrm{~Hz}, 1 \mathrm{H}), 1.49$ $1.58(\mathrm{~m}, 2 \mathrm{H}), 1.60$ (br s, 1H), 1.73 (ddt, $J=13.7 \mathrm{~Hz}, 4.6 \mathrm{~Hz}, 3.1 \mathrm{~Hz}, 1 \mathrm{H}), 1.86-1.96$ (m, 2H), $2.03-$ $2.13(\mathrm{~m}, 1 \mathrm{H}), 2.14-2.23(\mathrm{~m}, 2 \mathrm{H}), 2.24-2.32(\mathrm{~m}, 1 \mathrm{H}), 2.45(\mathrm{~s}, 1 \mathrm{H}) 3.72(\mathrm{~s}, 1 \mathrm{H}), 5.01-5.10(\mathrm{~m}, 2 \mathrm{H})$, 
5.78-5.88 (m, 1H); ${ }^{13} \mathrm{C}$ NMR $\left(150 \mathrm{MHz}, \mathrm{CDCl}_{3}\right): \delta 13.77\left(\mathrm{CH}_{3}\right), 15.80\left(\mathrm{CH}_{3}\right), 22.26\left(\mathrm{CH}_{2}\right), 24.12$ $\left(\mathrm{CH}_{2}\right), 31.65\left(\mathrm{CH}_{2}\right), 33.47(\mathrm{CH}), 37.53\left(\mathrm{CH}_{2}\right), 39.86\left(\mathrm{CH}_{2}\right), 43.33(\mathrm{CH}), 46.55(\mathrm{CH}), 67.97(\mathrm{CH})$, $68.55(\mathrm{C}), 72.33(\mathrm{C}), 116.09\left(\mathrm{CH}_{2}\right), 137.86(\mathrm{CH})$; IR (ATR): v = 3517, 3075, 2961, 2929, 2867, 1733, 1640, 1473, 1449, 1416, 1394, 1334, 1269, 1164, 1106, 1087, 1057, 1020, 994, 970, 909, 882, 849, 787, 755, 664, $639 \mathrm{~cm}^{-1}$; GC-MS (EI): m/z = 235 (1), 221 (2), 218 (2), 205 (10), 193 (15), 178 (14), 175 (21), 160 (10), 137 (22), 135 (19), 133 (12), 125 (14), 123 (15), 111 (14), 109 (24), 107 (20), 105 (16), 97 (20), 96 (10), 95 (32), 93 (24), 91 (25), 83 (20), 81 (42), 79 (29), 77 (19), 71 (11), 69 (20), 67 (30), 57 (11), 55 (47), 53 (20), 43 (100), 41 (64), 39 (27); anal. calcd for $\mathrm{C}_{15} \mathrm{H}_{24} \mathrm{O}_{2}: \mathrm{C}, 76.23 ; \mathrm{H}, 10.24$; found: C, 76.56; H, 10.04 .

\section{Epoxy ketone 17 by oxidation of epoxy alcohol 20}

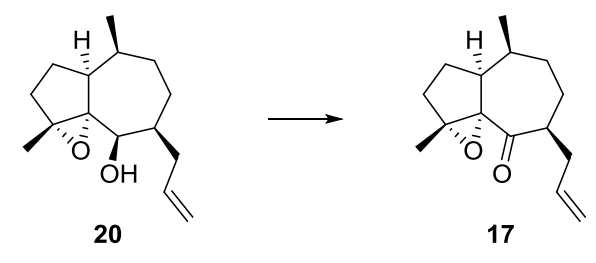

A solution of alcohol $20(11.9 \mathrm{mg}, 50.4 \mu \mathrm{mol})$ and PDC $(56.5 \mathrm{mg}, 150 \mu \mathrm{mol})$ in DMF (0.6 mL) was stirred at room temperature overnight. After TLC indicated complete conversion of $\mathbf{2 0}$, water $(2 \mathrm{~mL})$ was added, and the aqueous layer was extracted with ethyl acetate. The combined organic layers were washed with saturated aqueous $\mathrm{NaCl}$ and dried over magnesium sulfate. Column chromatography (pentane/ethyl acetate, 10/1) yielded ketone $17(11.8 \mathrm{mg}, 100 \%)$ as a colorless solid. For characterization data, see preparation of $\mathbf{1 7}$ by oxidation of alcohol $\mathbf{1 6}$.

\section{Epoxy lactone 22}

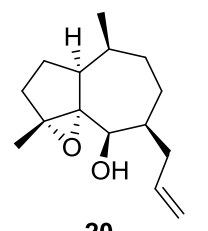

20

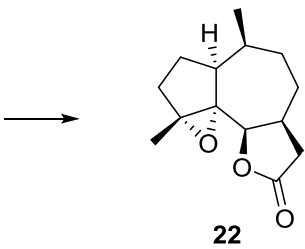

22

A solution of epoxy alcohol $20(51.0 \mathrm{mg}, 215.8 \mu \mathrm{mol})$ in acetone/water $(2.4 \mathrm{~mL}, 10 / 1)$ was treated with 2,6-lutidine $(50 \mu \mathrm{L}, 431.7 \mu \mathrm{mol}), N$-methylmorpholine $N$-oxide $(37.9 \mathrm{mg}, 323.5 \mu \mathrm{mol})$ and osmium tetroxide ( $22 \mu \mathrm{l}, 4.3 \mu \mathrm{mol}, 0.196 \mathrm{M}$ in toluene). This mixture was stirred for $5 \mathrm{~h} 50 \mathrm{~min}$ at room temperature. After complete conversion of $\mathbf{2 0}$, the resultant diol was oxidized by addition of PIDA $(104.3 \mathrm{mg}, 323.8 \mu \mathrm{mol})$ and stirring for additional $110 \mathrm{~min}$. Saturated aqueous sodium thiosulfate $(3 \mathrm{~mL})$ and water $(3 \mathrm{~mL})$ were added, and the aqueous layer was extracted three times with 
ethyl acetate. After washing the combined organic layers with saturated aqueous copper sulfate and drying over magnesium sulfate, the solvents were removed under reduced pressure, and the residue was dissolved in $\mathrm{CH}_{2} \mathrm{Cl}_{2}(2 \mathrm{~mL})$. PDC (324.7 mg, $\left.863.1 \mu \mathrm{mol}\right)$ was added, and the mixture was stirred at room temperature overnight. After filtration of the mixture over a pad of florisil (elution with ethyl acetate), column chromatography (pentane/ethyl acetate, 1/1) gave lactone 22 (47.3 $\mathrm{mg}, 93 \%)$ as colorless crystals.

$\mathrm{R}_{\mathrm{f}}=0.44$ (pentane/ethyl acetate $\left.1 / 1\right) ; \mathrm{mp} 67.6{ }^{\circ} \mathrm{C} ;[\alpha]_{\mathrm{D}}{ }^{21}-23.2\left(c 1.42, \mathrm{CHCl}_{3}\right) ;{ }^{1} \mathrm{H} \mathrm{NMR}(600 \mathrm{MHz}$, $\left.\mathrm{CDCl}_{3}\right): \delta 0.90(\mathrm{~d}, J=6.6 \mathrm{~Hz}, 3 \mathrm{H}), 1.37-1.47(\mathrm{~m}, 1 \mathrm{H}) 1.50(\mathrm{~s}, 3 \mathrm{H}), 1.53-1.67(\mathrm{~m}, 4 \mathrm{H}), 1.75-1.85(\mathrm{~m}$, 2H), 1.85-1.94 (m, 1H), 2.00 (ddd, $J=14.1 \mathrm{~Hz}, 9.4 \mathrm{~Hz}, 4.3 \mathrm{~Hz}, 1 \mathrm{H}), 2.10-2.21(\mathrm{~m}, 1 \mathrm{H}) 2.37$ (dt, $J=$ $9.3 \mathrm{~Hz}, 4.5 \mathrm{~Hz}, 1 \mathrm{H}), 2.77-2.97(\mathrm{~m}, 2 \mathrm{H}), 5.16(\mathrm{~d}, J=7.9 \mathrm{~Hz}, 1 \mathrm{H}) ;{ }^{13} \mathrm{C} \mathrm{NMR}\left(125 \mathrm{MHz}, \mathrm{CDCl}_{3}\right): \delta$ $16.60\left(\mathrm{CH}_{3}\right), 19.64\left(\mathrm{CH}_{3}\right), 21.58\left(\mathrm{CH}_{2}\right), 29.21\left(\mathrm{CH}_{2}\right), 29.49\left(\mathrm{CH}_{2}\right), 30.77(\mathrm{CH}), 33.14\left(\mathrm{CH}_{2}\right), 34.07$ $(\mathrm{CH}), 35.56\left(\mathrm{CH}_{2}\right), 47.73(\mathrm{CH}), 71.18(\mathrm{C}), 72.12(\mathrm{C}), 79.06(\mathrm{CH}), 175.81(\mathrm{C})$; IR (ATR): $v$ = 3018, 2953, 2928, 2871, 2056, 1867, 1843, 1778, 1650, 1458, 1413, 1375, 1335, 1256, 1186, 1112, 1040, 984, 943, 866, 818, $720 \mathrm{~cm}^{-1}$; GC-MS (EI): m/z = 236 (3), 235 (3), 221 (3), 208 (1), 194 (4), 192 (1), 178 (22), 163 (9), 149 (10), 135 (13), 134 (20), 133 (24), 123 (11), 121 (15), 119 (35), 109 (14), 107 (25), 106 (15), 105 (26), 97 (11), 95 (39), 94 (60), 93 (34), 92 (14), 91 (35), 82 (11), 81 (30), 79 (37), 77 (22), 69 (16), 67 (30), 65 (10), 55 (53), 43 (100); anal. calcd for $\mathrm{C}_{14} \mathrm{H}_{20} \mathrm{O}_{3}$ : C, 71.16; H, 8.53; found: C, 70.98; H, 8.61.

\section{$\underline{\text { Enamino lactone } 24}$}
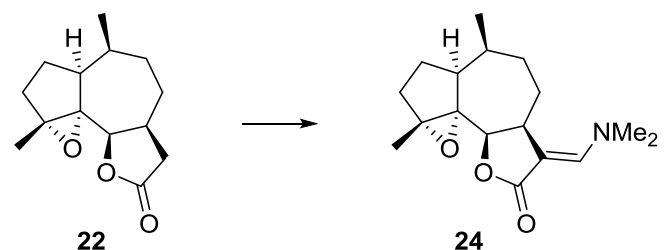

Lactone $22(67.0 \mathrm{mg}, 283.5 \mu \mathrm{mol})$ was treated with Bredereck's reagent 23 (480 $\mu \mathrm{L}, 2.32 \mathrm{mmol})$. After stirring for $4.5 \mathrm{~h}$ at $90{ }^{\circ} \mathrm{C}$, the volatile components were evaporated under reduced pressure, and the residue was dissolved in methanol $(1.4 \mathrm{~mL})$ and $\mathrm{K}_{2} \mathrm{CO}_{3}$ solution $(1.4 \mathrm{~mL}, 20 \mathrm{wt} \%$ in water). This mixture was stirred for $1 \mathrm{~h}$ under reflux, and then $\mathrm{NaH}_{2} \mathrm{PO}_{4}(2.8 \mathrm{~mL}, 50 \mathrm{wt} \%$ in water) and water (3 $\mathrm{mL}$ ) were added. The aqueous layer was extracted with ethyl acetate, and the combined organic layers were dried over magnesium sulfate. Column chromatographic purification (ethyl acetate) furnished enamine 24 (78.3 $\mathrm{mg}, 95 \%)$ as a slightly yellow solid.

$\mathrm{R}_{\mathrm{f}}=0.24$ (ethyl acetate); mp $197.8{ }^{\circ} \mathrm{C} ;[\alpha]_{\mathrm{D}}{ }^{22}-7.6\left(c 1.60, \mathrm{CHCl}_{3}\right) ;{ }^{1} \mathrm{H} \mathrm{NMR}\left(300 \mathrm{MHz}, \mathrm{CDCl}_{3}\right): \delta$ $0.91(\mathrm{~d}, J=6.8 \mathrm{~Hz}, 3 \mathrm{H}), 1.34-1.45(\mathrm{~m}, 1 \mathrm{H}), 1.56(\mathrm{~s}, 3 \mathrm{H}), 1.59-1.84(\mathrm{~m}, 6 \mathrm{H}), 1.91-2.08(\mathrm{~m}, 2 \mathrm{H}), 2.40$ 
$(\mathrm{dt}, J=8.8 \mathrm{~Hz}, 4.4 \mathrm{~Hz}, 1 \mathrm{H}), 3.03(\mathrm{~s}, 6 \mathrm{H}), 3.56(\mathrm{t}, J=9.6 \mathrm{~Hz}, 1 \mathrm{H}), 5.01(\mathrm{~d}, J=8.5 \mathrm{~Hz}, 1 \mathrm{H}), 7.14(\mathrm{~d}, J$ $=1.5 \mathrm{~Hz}, 1 \mathrm{H}) ;{ }^{13} \mathrm{C}$ NMR $\left(75 \mathrm{MHz}, \mathrm{CDCl}_{3}\right): \delta 16.43\left(\mathrm{CH}_{3}\right), 19.87\left(\mathrm{CH}_{3}\right), 20.60\left(\mathrm{CH}_{2}\right), 28.79\left(\mathrm{CH}_{2}\right)$, $29.87\left(\mathrm{CH}_{2}\right), 30.30(\mathrm{CH}), 33.60\left(\mathrm{CH}_{2}\right), 37.79(\mathrm{CH}), 42.00\left(2 \mathrm{C}, \mathrm{CH}_{3}\right), 47.12(\mathrm{CH}), 71.55(\mathrm{C}), 72.49$ (C), $76.18(\mathrm{CH}), 92.30(\mathrm{C}), 147.65(\mathrm{CH}), 173.80$ (C); IR (ATR): v = 3016, 2949, 2913, 2882, 2842, 2801, 2133, 2052, 1840, 1790, 1771, 1708, 1651, 1609, 1507, 1475, 1415, 1377, 1318, 1282, 1264, 1198, 1126, 1069, 1038, 1012, 983, 937, 891, 949, 813, 755, 731, 689, $625 \mathrm{~cm}^{-1}$. GC-MS (EI): m/z = 291 (72), 276 (17), 262 (23), 248 (14), 246 (12), 235 (31), 234 (33), 220 (21), 206 (20), 192 (18), 178 (21), 166 (38), 139 (83), 134 (13), 111 (20), 98 (20), 94 (23), 91 (19), 82 (100), 79 (13), 67 (14), 58 (15), 55 (20), 53 (19), 43 (39); HRMS (ESI-TOF): m/z calcd for $\mathrm{C}_{17} \mathrm{H}_{26} \mathrm{NO}_{3}[\mathrm{M}+\mathrm{H}]^{+}: 292.1913$; found: 292.1910.

$\underline{\alpha-M e t h y l e n e ~ l a c t o n e ~} 3$
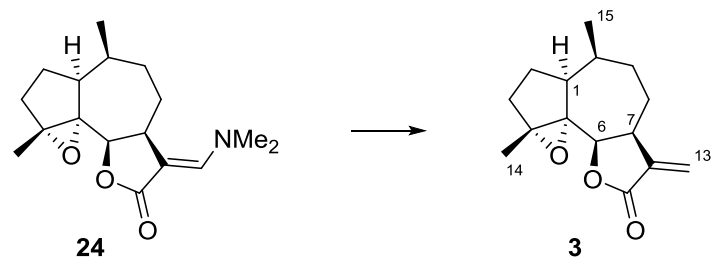

To a solution of enamine $24(339.2 \mathrm{mg}, 1.164 \mathrm{mmol})$ in THF $(28 \mathrm{~mL})$ at $-78{ }^{\circ} \mathrm{C}$ was added DIBAL (2.8 mL, $2.8 \mathrm{mmol}, 1 \mathrm{M}$ in toluene). After stirring the resultant mixture for $1 \mathrm{~h}$ at room temperature, saturated aqueous $\mathrm{NH}_{4} \mathrm{Cl}(3.5 \mathrm{~mL})$ and water $(5 \mathrm{~mL})$ were added. The aqueous layer was extracted with ethyl acetate, and the organic layer was dried over magnesium sulfate. Flash chromatography (pentane/ethyl acetate, $3 / 1)$ yielded $\alpha$-methylene lactone $\mathbf{3}(240.9 \mathrm{mg}, 83 \%)$ as a colorless oil. ${ }^{3}$

$\mathrm{R}_{\mathrm{f}}=0.24$ (pentane/ethyl acetate, $\left.3 / 1\right) ;[\alpha]_{\mathrm{D}}{ }^{25}-55.4\left(c 0.97, \mathrm{CHCl}_{3}\right) ;{ }^{1} \mathrm{H}$ NMR $\left(600 \mathrm{MHz}, \mathrm{CDCl}_{3}\right): \delta$ $0.92(\mathrm{~d}, J=6.8 \mathrm{~Hz}, 3 \mathrm{H}), 1.40(\mathrm{~s}, 3 \mathrm{H}), 1.43-1.50(\mathrm{~m}, 1 \mathrm{H}), 1.53-1.68(\mathrm{~m}, 4 \mathrm{H}), 1.74$ (ddd, $J=14.5 \mathrm{~Hz}$, $10.5 \mathrm{~Hz}, 7.0 \mathrm{~Hz}, 1 \mathrm{H}), 1.87-1.95$ (m, 2H), 1.99 (ddd, $J=14.4 \mathrm{~Hz}, 9.3 \mathrm{~Hz}, 4.5 \mathrm{~Hz}, 1 \mathrm{H}), 2.38$ (ddd, $J=$ $9.4 \mathrm{~Hz}, 4.7 \mathrm{~Hz}, 4.7 \mathrm{~Hz}, 1 \mathrm{H}), 3.27-3.35(\mathrm{~m}, 1 \mathrm{H}), 5.21(\mathrm{~d}, J=9.0 \mathrm{~Hz}, 1 \mathrm{H}), 5.62(\mathrm{~d}, J=3.0 \mathrm{~Hz}, 1 \mathrm{H})$, $6.34(\mathrm{~d}, J=3.0 \mathrm{~Hz}, 1 \mathrm{H}) ;{ }^{13} \mathrm{C} \mathrm{NMR}\left(150 \mathrm{MHz}, \mathrm{CDCl}_{3}\right): \delta 16.48\left(\mathrm{CH}_{3}\right), 20.08\left(\mathrm{CH}_{3}\right), 20.85\left(\mathrm{CH}_{2}\right)$, $29.06\left(\mathrm{CH}_{2}\right), 29.68\left(\mathrm{CH}_{2}\right), 30.87(\mathrm{CH}), 33.12\left(\mathrm{CH}_{2}\right), 38.64(\mathrm{CH}), 47.63(\mathrm{CH}), 71.26(\mathrm{C}), 72.10(\mathrm{C})$, $76.39(\mathrm{CH}), 123.27\left(\mathrm{CH}_{2}\right), 138.55(\mathrm{C}), 169.53(\mathrm{C})$; IR (ATR): $v=2953,2925,2874,2137,2056,2030$, 2010, 1918, 1844, 1761, 1700, 1653, 1462, 1398, 1378, 1329, 1270, 1197, 1134, 1099, 1037, 1018, 988, 942, 864, 815, 709, 663, $624 \mathrm{~cm}^{-1}$; GC-MS (EI): m/z = 248 (5), 247 (5), 230 (5), 202 (7), 190 (33), 177 (45), 175 (12), 161 (16), 159 (14), 151 (13), 149 (12), 146 (36), 133 (29), 131 (54), 129 (10), 125 (18), 123 (28), 119 (31), 117 (24), 109 (13), 107 (23), 105 (47), 94 (100), 93 (33), 91 (50), 81 (27), 79 (51), 77 (30), 69 (12), 67 (23), 65 (15), 55 (37), 53 (40), 43 (83), 41 (44), 39 (28); HRMS (ESI- 
TOF): $\mathrm{m} / \mathrm{z}$ calcd for $\mathrm{C}_{15} \mathrm{H}_{21} \mathrm{O}_{3}[\mathrm{M}+\mathrm{H}]^{+}: 249.1485 ; \mathrm{C}_{15} \mathrm{H}_{24} \mathrm{NO}_{3}\left[\mathrm{M}+\mathrm{NH}_{4}\right]^{+}:$266.1751; found: 249.1489; 266.1751 .

Table 1: Chemical shifts [ppm] of synthetic epoxide $3\left(600 \mathrm{MHz}, \mathrm{CDCl}_{3}\right)$ and epoxide 3 derived from natural 1 by epoxidation ${ }^{3}\left(100 \mathrm{MHz}, \mathrm{CDCl}_{3}\right)$ in the absence and presence of $\mathrm{Eu}(\mathrm{fod})_{3}$.

\begin{tabular}{lllll}
\hline & Synthetic 3 & \multicolumn{3}{c}{ 3 from natural 1 } \\
\hline & & $+20 \mathrm{~mol} \% \mathrm{Eu}(\mathrm{fod})_{3}$ & $+\mathrm{Eu}(\mathrm{fod})_{3}$ \\
& & $\rightarrow \Delta[\mathrm{ppm}]$ & $\rightarrow \Delta[\mathrm{ppm}]$ \\
\hline $15-\mathrm{H}$ & $0.92(\mathrm{~d}, J=6.8 \mathrm{~Hz})$ & 0.15 & $0.92(\mathrm{~d}, J=6.5 \mathrm{~Hz})$ & 0.12 \\
$14-\mathrm{H}$ & $1.40(\mathrm{~s})$ & 1.24 & $1.39(\mathrm{~s})$ & 1.20 \\
$13-\mathrm{H}$ & $5.62(\mathrm{~d}, J=3.0 \mathrm{~Hz})$ & 0.46 & $5.60(\mathrm{~d}, J=2 \mathrm{~Hz})$ & 0.43 \\
$13-\mathrm{H}^{\prime}$ & $6.34(\mathrm{~d}, J=3.0 \mathrm{~Hz})$ & 0.73 & $6.31(\mathrm{~d}, J=3 \mathrm{~Hz})$ & 0.74 \\
$7-\mathrm{H}$ & $3.31(\mathrm{~m})$ & 0.62 & $3.30(\mathrm{~m})$ & 0.58 \\
$6-\mathrm{H}$ & $5.21(\mathrm{~d}, J=9.0 \mathrm{~Hz})$ & 0.99 & $5.18(\mathrm{~d}, J=8 \mathrm{~Hz})$ & 0.94 \\
$1-\mathrm{H}$ & $2.38(\mathrm{ddd}, J=9.4$ & 0.65 & $2.35(\mathrm{~m})$ & 0.64 \\
& $\mathrm{~Hz}, 4.7 \mathrm{~Hz}, 4.7 \mathrm{~Hz})$ & & & \\
\hline
\end{tabular}

\section{Epoxy lactone 5}

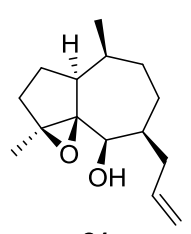

21

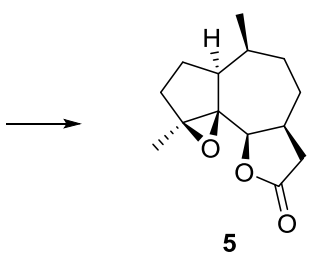

5

A solution of epoxy alcohol $21(31.4 \mathrm{mg}, 133 \mu \mathrm{mol})$ in acetone/water $(2.4 \mathrm{~mL}, 10 / 1)$ was treated with 2,6-lutidine $(31 \mu \mathrm{L}, 268 \mu \mathrm{mol}), N$-methylmorpholine $N$-oxide $(23.3 \mathrm{mg}, 199 \mu \mathrm{mol})$ and osmium tetroxide ( $14 \mu \mathrm{l}, 2.7 \mu \mathrm{mol}, 0.196 \mathrm{M}$ in toluene). This mixture was stirred for $5 \mathrm{~h}$ at room temperature. After complete conversion of 21, the resultant diol was oxidized by addition of PIDA (95.6 mg, 297 $\mu \mathrm{mol})$ and stirring for additional $75 \mathrm{~min}$. Saturated aqueous sodium thiosulfate (1 mL) and water (1 $\mathrm{mL}$ ) were added, and the aqueous layer was extracted three times with ethyl acetate. After washing the combined organic layers with saturated aqueous copper sulfate and drying over magnesium sulfate, the solvents were removed under reduced pressure, and the residue was dissolved in $\mathrm{CH}_{2} \mathrm{Cl}_{2}(2 \mathrm{~mL})$. PIDA ( $85.5 \mathrm{mg}, 266 \mu \mathrm{mol})$ and TEMPO $(4.15 \mathrm{mg}, 26.6 \mu \mathrm{mol})$ were added, and the mixture was stirred at room temperature overnight. After complete conversion was indicated by TLC, saturated aqueous sodium thiosulfate $(1 \mathrm{~mL})$ and water $(1 \mathrm{~mL})$ were added. The aqueous layer was extracted with ethyl acetate, and the combined organic layers were dried over magnesium sulfate. 
Chromatographic purification (pentane/ethyl acetate, 1/1) provided lactone $5(23.7 \mathrm{mg}, 76 \%)$ as a white solid.

$\mathrm{R}_{\mathrm{f}}=0.68$ (pentane/ethyl acetate, $\left.1 / 1\right) ; \mathrm{mp} 98-99{ }^{\circ} \mathrm{C} ;[\alpha]_{\mathrm{D}}{ }^{21}-24.6\left(c 0.85, \mathrm{CHCl}_{3}\right) ;{ }^{1} \mathrm{H} \mathrm{NMR}(500 \mathrm{MHz}$, $\left.\mathrm{CDCl}_{3}\right): \delta 0.99(\mathrm{~d}, J=6.6 \mathrm{~Hz}, 3 \mathrm{H}), 1.34-1.49(\mathrm{~m}, 3 \mathrm{H}), 1.51(\mathrm{~s}, 3 \mathrm{H}), 1.60(\mathrm{~m}, 1 \mathrm{H}), 1.69-1.76(\mathrm{~m}, 1 \mathrm{H})$, $1.80(\mathrm{~m}, 1 \mathrm{H}), 1.90(\mathrm{dd}, J=13.6 \mathrm{~Hz}, 7.6 \mathrm{~Hz}, 1 \mathrm{H}), 1.98-2.15(\mathrm{~m}, 3 \mathrm{H}), 2.32(\mathrm{dd}, J=17.7 \mathrm{~Hz}, 7.6 \mathrm{~Hz}$, $1 \mathrm{H}), 2.64(\mathrm{dd}, J=17.5 \mathrm{~Hz}, 9.0 \mathrm{~Hz}, 1 \mathrm{H}), 2.82-2.93(\mathrm{~m}, 1 \mathrm{H}), 4.92(\mathrm{~d}, J=7.9 \mathrm{~Hz}, 1 \mathrm{H}) ;{ }^{13} \mathrm{C}$ NMR $(125$ $\left.\mathrm{MHz}, \mathrm{CDCl}_{3}\right): \delta 16.42\left(\mathrm{CH}_{3}\right), 16.74\left(\mathrm{CH}_{3}\right), 22.10\left(\mathrm{CH}_{2}\right), 25.16\left(\mathrm{CH}_{2}\right), 31.15\left(\mathrm{CH}_{2}\right), 31.35(\mathrm{CH}), 32.65$ $\left(\mathrm{CH}_{2}\right), 36.02\left(\mathrm{CH}_{2}\right), 38.68(\mathrm{CH}), 45.28(\mathrm{CH}), 67.63(\mathrm{C}), 69.97(\mathrm{C}), 79.19(\mathrm{CH}), 176.32(\mathrm{C})$; IR (ATR): $v=2957,2929,2872,2137,2056,2030,2009,1867,1843,1756,1684,1651,1635,1457,1422,1381$, 1321, 1293, 1206, 1164, 1150, 1084, 1044, 1018, 1000, 941, 921, 881, 858, 819, 798, 762, 731, 697, 649, $618 \mathrm{~cm}^{-1}$; GC-MS (EI): m/z = 236 (4), 235 (4), 221 (3), 193 (4), 192 (4), 178 (23), 163 (9), 151 (10), 150 (12), 149 (12), 135 (14), 134 (23), 133 (27), 123 (12), 121 (15), 119 (37), 109 (14), 107 (28), 106 (16), 105 (27), 97 (13), 95 (41), 94 (56), 93 (36), 92 (14), 91 (36), 83 (10), 82 (11), 81 (33), 79 (41), 77 (23), 69 (17), 67 (32), 65 (11), 55 (60), 53 (26), 43 (100); HRMS (EI): m/z calcd for $\mathrm{C}_{14} \mathrm{H}_{20} \mathrm{O}_{3}$ $[\mathrm{M}+\mathrm{H}]^{+}:$236.1412; found: 236.1397 .

\section{$\underline{\text { Enamino lactone } \mathbf{S 1}}$}
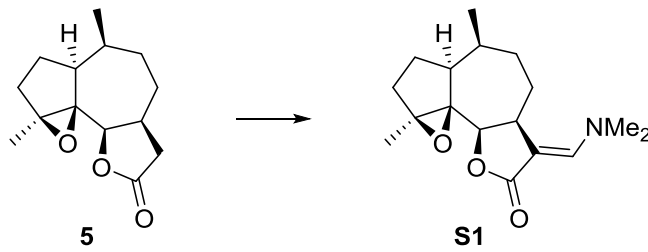

Lactone 5 (43.6 mg, $184.5 \mu \mathrm{mol})$ was treated with Bredereck's reagent $23(314.3 \mu \mathrm{L}, 1.52 \mathrm{mmol})$. After stirring for $4 \mathrm{~h}$ at $90^{\circ} \mathrm{C}$, the volatile components were evaporated under reduced pressure, and the residue was dissolved in methanol $(0.92 \mathrm{~mL})$ and $\mathrm{K}_{2} \mathrm{CO}_{3}$ solution $(0.92 \mathrm{~mL}, 20 \mathrm{wt} \%$ in water). This mixture was stirred for $1 \mathrm{~h}$ under reflux, and then $\mathrm{NaH}_{2} \mathrm{PO}_{4}(1.8 \mathrm{~mL}, 50 \mathrm{wt} \%$ in water) and water $(2 \mathrm{~mL})$ were added. The aqueous layer was extracted with ethyl acetate, and the combined organic layers were dried over magnesium sulfate. Flash chromatography (ethyl acetate) gave enamine S1 $(48.7 \mathrm{mg}, 91 \%)$ as a slightly yellow solid.

$\mathrm{R}_{\mathrm{f}}=0.22$ (ethyl acetate); ${ }^{1} \mathrm{H} \mathrm{NMR}\left(500 \mathrm{MHz}, \mathrm{CDCl}_{3}\right): \delta 0.95(\mathrm{~d}, J=6.6 \mathrm{~Hz}, 3 \mathrm{H}), 1.23-1.46(\mathrm{~m}, 2 \mathrm{H})$, 1.47-1.54 (m, 2H), 1.55 (s, 3H), 1.57-1.60 (m, 1H), 1.72-1.80 (m, 1H), $1.92(\mathrm{dd}, J=13.7 \mathrm{~Hz}, 7.7 \mathrm{~Hz}$, 1H), 2.10-2.20 (m, 2H), 2.20-2.29 (m, 1H), $3.04(\mathrm{~s}, 6 \mathrm{H}), 3.56(\mathrm{dd}, J=5.7 \mathrm{~Hz}, 3.8 \mathrm{~Hz}, 1 \mathrm{H}), 4.71(\mathrm{~d}, J$ $=6.9 \mathrm{~Hz}, 1 \mathrm{H}), 7.12(\mathrm{~d}, J=1.3 \mathrm{~Hz}, 1 \mathrm{H}) ;{ }^{13} \mathrm{C} \mathrm{NMR}\left(125 \mathrm{MHz}, \mathrm{CDCl}_{3}\right): \delta 15.43\left(\mathrm{CH}_{3}\right), 17.36\left(\mathrm{CH}_{3}\right)$, $21.35\left(\mathrm{CH}_{2}\right), 28.19\left(\mathrm{CH}_{2}\right), 30.37\left(\mathrm{CH}_{2}\right), 30.83(\mathrm{CH}), 32.58\left(\mathrm{CH}_{2}\right), 40.75(\mathrm{CH}), 41.85\left(2 \mathrm{C}, \mathrm{CH}_{3}\right), 44.97$ 
(CH), $65.93(\mathrm{C}), 68.63(\mathrm{C}), 75.55(\mathrm{CH}), 93.76(\mathrm{C}), 146.56(\mathrm{CH}), 174.25(\mathrm{C}) ; \mathrm{GC}-\mathrm{MS}(\mathrm{EI}): \mathrm{m} / \mathrm{z}=291$ (85), 262 (33), 235 (43), 234 (28), 220 (20), 207 (17), 204 (14), 192 (20), 178 (23), 176 (15), 166 (31), 165 (15), 164 (12), 152 (14), 150 (11), 148 (11), 140 (16), 139 (50), 138 (17), 134 (10), 122 (10), 111 (19), 110 (12), 105 (12), 98 (19), 97 (25), 95 (14), 94 (22), 91 (18), 85 (68), 84 (10), 83 (10), 82 (100), 79 (13), 77 (13), 71 (12), 67 (15), 58 (17), 57 (21), 55 (19), 53 (12), 44 (13), 43 (37), 42 (36), 41 (42), $39(13)$.

$\underline{\alpha-M e t h y l e n e ~ l a c t o n e ~} 2$ by DIBAL reduction of enamino lactone $\mathbf{S 1}$
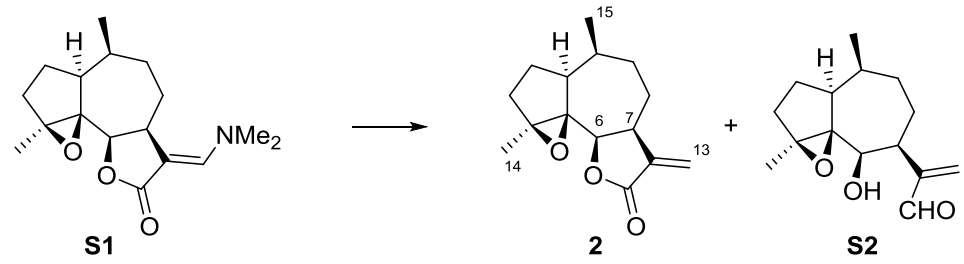

To a solution of enamine $\mathbf{S 1}(44.3 \mathrm{mg}, 152 \mu \mathrm{mol})$ in THF $(3.2 \mathrm{~mL})$ at $-78{ }^{\circ} \mathrm{C}$ was added DIBAL (370 $\mu \mathrm{L}, 370 \mu \mathrm{mol}, 1 \mathrm{M}$ in toluene). After stirring the resultant mixture for $1 \mathrm{~h}$ at room temperature, saturated aqueous $\mathrm{NH}_{4} \mathrm{Cl}(460 \mu \mathrm{L})$ and magnesium sulfate were added. The suspension was filtered (elution with ethyl acetate). Flash chromatography (pentane/diethyl ether, 3/2) yielded $\alpha$-methylene lactone $2(16.7 \mathrm{mg}, 44 \%)$ as a white solid ${ }^{3}$ and the byproduct $\mathbf{S 2}(4.8 \mathrm{mg}, 13 \%)$ as a colorless oil. Compound $\mathbf{S 2}$ was converted into lactone $\mathbf{2}$ after standing in air for several months.

2: $\mathrm{R}_{\mathrm{f}}=0.30$ (pentane/diethyl ether, 3/2); $\mathrm{mp} 85.4{ }^{\circ} \mathrm{C}\left\{\right.$ ref 3: $\left.\mathrm{mp} 119.4{ }^{\circ} \mathrm{C}\right\} ;[\alpha]_{\mathrm{D}}{ }^{22}-85.1(c 0.52$, $\left.\mathrm{CHCl}_{3}\right)\left\{\operatorname{ref} 3:[\alpha]_{\mathrm{D}}{ }^{24}-203\left(c 0.8, \mathrm{CHCl}_{3}\right)\right\} ;{ }^{1} \mathrm{H} \mathrm{NMR}\left(600 \mathrm{MHz}, \mathrm{CDCl}_{3}\right): \delta 0.96(\mathrm{~d}, J=6.8 \mathrm{~Hz}, 3 \mathrm{H})$, $1.31-1.37$ (m, 1H), 1.39-1.46 (m, 1H), 1.57 (s, 3H), 1.58-1.62 (m, 2H), 1.75-1.85 (m, 2H), 1.90 (dd, J $=13.9 \mathrm{~Hz}, 7.9 \mathrm{~Hz}, 1 \mathrm{H}), 2.07-2.16(\mathrm{~m}, 2 \mathrm{H}), 2.20-2.29(\mathrm{~m}, 1 \mathrm{H}), 3.38-3.45(\mathrm{~m}, 1 \mathrm{H}), 5.00(\mathrm{~d}, J=8.3 \mathrm{~Hz}$, $1 \mathrm{H}), 5.57(\mathrm{~d}, J=2.5 \mathrm{~Hz}, 1 \mathrm{H}), 6.25(\mathrm{~d}, J=2.5 \mathrm{~Hz}, 1 \mathrm{H}) ;{ }^{13} \mathrm{C} \mathrm{NMR}\left(150 \mathrm{MHz}, \mathrm{CDCl}_{3}\right): \delta 16.16\left(\mathrm{CH}_{3}\right)$, $17.70\left(\mathrm{CH}_{3}\right), 20.71\left(\mathrm{CH}_{2}\right), 26.75\left(\mathrm{CH}_{2}\right), 29.58\left(\mathrm{CH}_{2}\right), 30.89(\mathrm{CH}), 32.93\left(\mathrm{CH}_{2}\right), 41.56(\mathrm{CH}), 45.21$ $(\mathrm{CH}), 67.55(\mathrm{C}), 69.91(\mathrm{C}), 76.94(\mathrm{CH}), 121.50\left(\mathrm{CH}_{2}\right), 139.46(\mathrm{C}), 169.72(\mathrm{C})$; IR (ATR): $v=3490$, 3014, 2959, 2942, 2919, 2869, 2849, 2135, 2056, 2030, 2009, 1868, 1843, 1827, 1755, 1716, 1698, 1684, 1653, 1634, 1455, 1428, 1404, 1377, 1344, 1324, 1272, 1237, 1196, 1149, 1101, 1064, 1033, 1011, 988, 956, 935, 918, 874, 813, 793, 770, 677, 663, 650, $628 \mathrm{~cm}^{-1}$; GC-MS (EI): m/z = 248 (6), 247 (3), 191 (11), 190 (28), 175 (11), 161 (14), 146 (30), 131 (50), 123 (33), 117 (21), 107 (22), 105 (44), 97 (12), 94 (99), 91 (52), 67 (32), 65 (17), 88 (44), 51 (11), 43 (100), 41 (59), 39 (39); HRMS (ESI-TOF): $\mathrm{m} / \mathrm{z}$ calcd for $\mathrm{C}_{15} \mathrm{H}_{21} \mathrm{O}_{3}[\mathrm{M}+\mathrm{H}]^{+}: 249.1485$; found: 249.1490 . 
Table 2: Chemical shifts [ppm] of synthetic 4,5-epoxyosmitopsin (2) (600 MHz, $\left.\mathrm{CDCl}_{3}\right)$ and natural 4,5-epoxyosmitopsin $(2)^{3}\left(100 \mathrm{MHz}, \mathrm{CDCl}_{3}\right)$ in the absence and presence of $\mathrm{Eu}(\mathrm{fod})_{3}$.

\begin{tabular}{lllll}
\hline & Synthetic 2 & \multicolumn{3}{c}{ Natural 2 } \\
\hline & & $+20 \mathrm{~mol} \% \mathrm{Eu}(\mathrm{fod})_{3}$ & $+\mathrm{Eu}(\mathrm{fod})_{3}$ \\
& & $\rightarrow \Delta[\mathrm{ppm}]$ & $\rightarrow \Delta[\mathrm{ppm}]$ \\
\hline $15-\mathrm{H}$ & $0.96(\mathrm{~d}, J=6.8 \mathrm{~Hz})$ & 0.04 & $1.04(\mathrm{~d}, J=6.5 \mathrm{~Hz})$ & 0.26 \\
$14-\mathrm{H}$ & $1.57(\mathrm{~s})$ & 0.09 & $1.34(\mathrm{~s})$ & 0.40 \\
$13-\mathrm{H}$ & $5.57(\mathrm{~d}, J=2.5 \mathrm{~Hz})$ & 0.10 & $5.62(\mathrm{~d}, J=1.8 \mathrm{~Hz})$ & 0.58 \\
$13-\mathrm{H}^{\prime}$ & $6.25(\mathrm{~d}, J=2.5 \mathrm{~Hz})$ & 0.24 & $6.31(\mathrm{~d}, J=2 \mathrm{~Hz})$ & 1.33 \\
$7-\mathrm{H}$ & $3.42(\mathrm{~m})$ & 0.11 & $3.32(\mathrm{~m})$ & 0.70 \\
$6-\mathrm{H}$ & $5.00(\mathrm{~d}, J=8.3 \mathrm{~Hz})$ & 0.13 & $5.01(\mathrm{~d}, J=7 \mathrm{~Hz})$ & 1.03 \\
\hline
\end{tabular}

S2: $\mathrm{R}_{\mathrm{f}}=0.22$ (pentane/diethyl ether, 3/2); ${ }^{1} \mathrm{H}$ NMR (500 MHz, $\left.\mathrm{CDCl}_{3}\right): \delta 1.06(\mathrm{~d}, J=7.3 \mathrm{~Hz}, 3 \mathrm{H}$ ), $1.19(\mathrm{~s}, 3 \mathrm{H}), 1.29-1.45(\mathrm{~m}, 3 \mathrm{H}), 1.62-1.72(\mathrm{~m}, 3 \mathrm{H}), 1.73-1.84(\mathrm{~m}, 4 \mathrm{H}), 1.96(\mathrm{td}, J=7.3 \mathrm{~Hz}, 3.8 \mathrm{~Hz}$, 1H), 2.35-2.42 (m, 1H), $3.11(\mathrm{dd}, J=11.3 \mathrm{~Hz}, 2.2 \mathrm{~Hz}, 1 \mathrm{H}), 6.13(\mathrm{~s}, 1 \mathrm{H}), 6.54(\mathrm{~s}, 1 \mathrm{H}), 9.57(\mathrm{~s}, 1 \mathrm{H})$; ${ }^{13} \mathrm{C}$ NMR $\left(150 \mathrm{MHz}, \mathrm{CDCl}_{3}\right): \delta 12.82\left(\mathrm{CH}_{3}\right), 24.39\left(\mathrm{CH}_{3}\right), 25.31\left(\mathrm{CH}_{2}\right), 25.57\left(\mathrm{CH}_{2}\right), 33.06(\mathrm{CH})$, $36.08\left(\mathrm{CH}_{2}\right), 36.81(\mathrm{CH}), 39.19\left(\mathrm{CH}_{2}\right), 41.99(\mathrm{CH}), 61.07(\mathrm{CH}), 72.72(\mathrm{C}), 75.81(\mathrm{C}), 134.62\left(\mathrm{CH}_{2}\right)$, 154.27 (C), 193.87 (CH); GC-MS (EI): m/z = 250 (1), 232 (1), 217 (6), 207 (4), 192 (8), 175 (19), 164 (17), 149 (18), 136 (22), 121 (25), 109 (44), 96 (68), 79 (38), 67 (30), 55 (48), 43 (100).

$\underline{\alpha-M e t h y l e n e ~ l a c t o n e ~} 2$ by alkylation of epoxy lactone $\mathbf{5}$

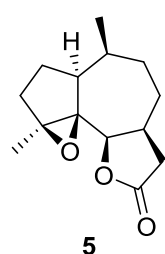

5

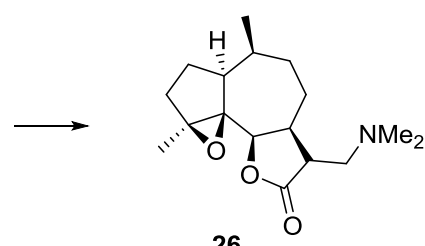

26

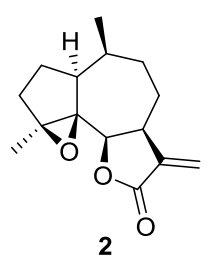

$2^{\circ}$

To a solution of hexamethyldisilazane $(701 \mu \mathrm{L}, 3.39 \mathrm{mmol})$ in $\mathrm{THF}(4.8 \mathrm{~mL})$ at $-78{ }^{\circ} \mathrm{C}$ was added $\operatorname{BuLi}\left(2.83 \mathrm{~mL}, 3.40 \mathrm{mmol}, 1.2 \mathrm{M}\right.$ in hexane). The mixture was warmed to $0{ }^{\circ} \mathrm{C}$ for $1 \mathrm{~h}$ and then cooled to $-78{ }^{\circ} \mathrm{C}$ again. A solution of lactone $5(70.9 \mathrm{mg}, 300 \mu \mathrm{mol})$ in THF (4.8 mL) was added dropwise to the LiHMDS solution at $-78^{\circ} \mathrm{C}$. After stirring at this temperature for $1.5 \mathrm{~h}$, Eschenmoser salt 25 (952.3 mg, $5.15 \mathrm{mmol}$ ) was added in a countercurrent of argon. The mixture was stirred for 30 min at $-78{ }^{\circ} \mathrm{C}$, then it was warmed to $-30{ }^{\circ} \mathrm{C}$, and stirring was continued for additional $4 \mathrm{~h}$. After complete conversion was indicated by TLC, the reaction was quenched through addition of aqueous $\mathrm{HCl}$ (2 M, until slightly acidic), $\mathrm{K}_{2} \mathrm{CO}_{3}$ (until slightly basic), and water $(5 \mathrm{~mL})$. The aqueous layer was extracted with ethyl acetate, and the organic layer was dried over magnesium sulfate. The solvents were removed under reduced pressure, and the residue was dissolved in methanol $(12.6 \mathrm{~mL})$ and 
treated with methyl iodide $(375 \mu \mathrm{L}, 6.02 \mathrm{mmol})$. After stirring at room temperature overnight, methanol was removed under reduced pressure. The residue was dissolved in 1,4-dioxane (12 mL) and treated with DBU $(90 \mu \mathrm{L}, 602 \mu \mathrm{mol})$. The mixture was stirred for $2 \mathrm{~h}$ at room temperature, then water $(10 \mathrm{~mL})$ was added, and the aqueous layer was extracted with ethyl acetate. After drying over magnesium sulfate, column chromatography (pentane/diethyl ether, 2/1) yielded $\alpha$-methylene lactone 2 (31.6 mg, 42\%) as a white solid. ${ }^{3} \mathrm{R}_{\mathrm{f}}=0.24$ (pentane/diethyl ether, 2/1). For further data, see preparation of $\mathbf{2}$ by DIBAL reduction of enamino lactone $\mathbf{S 1}$.

Epoxy alcohol 20 by reduction of epoxy ketone $\mathbf{1 7}$

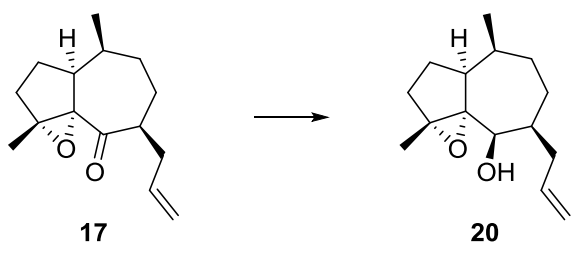

To a solution of epoxy ketone $17(129.6 \mathrm{mg}, 553.0 \mu \mathrm{mol})$ in THF $(23 \mathrm{~mL})$ cooled to $-78{ }^{\circ} \mathrm{C}$ was added dropwise L-selectride $(1.1 \mathrm{~mL}, 1.1 \mathrm{mmol}, 1 \mathrm{M}$ in THF), and then the mixture was warmed to room temperature over a period of $90 \mathrm{~min}$. After complete conversion was indicated by TLC, the mixture was cooled to $0{ }^{\circ} \mathrm{C}$, and $\mathrm{NaOH}\left(3.2 \mathrm{~mL}, 2 \mathrm{M}\right.$ in water) and $\mathrm{H}_{2} \mathrm{O}_{2}(2.2 \mathrm{~mL}, 30 \mathrm{wt} \%)$ diluted with water $(25 \mathrm{~mL})$ were added. The aqueous layer was extracted with dichloromethane, and the combined organic layers were washed with saturated aqueous $\mathrm{NaCl}$ and dried over magnesium sulfate. Flash chromatography (pentane/ethyl acetate, 20/1) provided epoxy alcohol 20 (127.1 $\mathrm{mg}, 97 \%$ ) as a white solid. For characterization data, see preparation of $\mathbf{2 0}$ by epoxidation of dienol 19.

\section{$\underline{\text { Unsaturated lactone } 27}$}

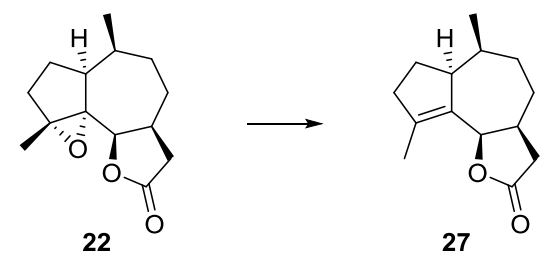

In a flame-dried round bottom flask $\mathrm{WCl}_{6}(79.3 \mathrm{mg}, 200 \mu \mathrm{mol})$ was dissolved in THF $(1.7 \mathrm{~mL})$. The solution was cooled to $-78{ }^{\circ} \mathrm{C}$ and mixed with $\mathrm{BuLi}(0.5 \mathrm{~mL}, 800 \mu \mathrm{mol}, 1.6 \mathrm{M}$ in hexane). After stirring for $10 \mathrm{~min}$ at this temperature, the mixture was warmed to ambient temperature and stirred for another hour. A spatula tip of LiI and a solution of $\mathbf{2 2}(23.6 \mathrm{mg}, 99.9 \mu \mathrm{mol})$ in THF $(1 \mathrm{~mL})$ were added. After $2 \mathrm{~h}$ at room temperature the mixture was diluted with aqueous Rochelle salt $(1 \mathrm{~mL}, 1.5$ M) and aqueous $\mathrm{NaOH}(1 \mathrm{~mL}, 2 \mathrm{M})$. The aqueous layer was extracted with diethyl ether, and the 
combined organic layers were washed with saturated aqueous $\mathrm{NaCl}$. After drying over magnesium sulfate, column chromatography (pentane/ethyl acetate, $4 / 1 \rightarrow 2 / 1$ ) afforded unsaturated lactone 27 (12.3 mg, 56\%, 83\% brsm) as a white solid and unreacted starting material 22 (7.6 mg).

$\mathrm{R}_{\mathrm{f}}=0.74$ (pentane/ethyl acetate, $\left.1 / 1\right) ; \mathrm{mp} 60.9^{\circ} \mathrm{C} ;[\alpha]_{\mathrm{D}}{ }^{22}-8.7\left(c 0.95, \mathrm{CHCl}_{3}\right) ;{ }^{1} \mathrm{H}$ NMR $(600 \mathrm{MHz}$, $\left.\mathrm{CDCl}_{3}\right): \delta 0.85(\mathrm{~d}, J=6.8 \mathrm{~Hz}, 3 \mathrm{H}), 1.40-1.46(\mathrm{~m}, 1 \mathrm{H}), 1.48-1.57(\mathrm{~m}, 3 \mathrm{H}), 1.67-1.76(\mathrm{~m}, 1 \mathrm{H}), 1.80(\mathrm{~s}$, $3 \mathrm{H}), 1.89-1.95(\mathrm{~m}, 1 \mathrm{H}), 1.95-2.01(\mathrm{~m}, 1 \mathrm{H}), 2.13(\mathrm{dd}, J=17.5 \mathrm{~Hz}, 4.0 \mathrm{~Hz}, 1 \mathrm{H}), 2.20-2.25(\mathrm{~m}, 1 \mathrm{H})$, 2.33-2.42 (m, 1H), 2.56-2.65 (m, 1H), 2.76 (dd, $J=17.3 \mathrm{~Hz}, 8.3 \mathrm{~Hz}, 1 \mathrm{H}), 2.80$ (br s., 1H), 5.24 (d, $J$ $=5.6 \mathrm{~Hz}, 1 \mathrm{H}) ;{ }^{13} \mathrm{C} \mathrm{NMR}\left(150 \mathrm{MHz}, \mathrm{CDCl}_{3}\right): \delta 15.25\left(\mathrm{CH}_{3}, 2 \mathrm{C}\right), 27.28\left(\mathrm{CH}_{2}\right), 27.49\left(\mathrm{CH}_{2}\right), 33.57$ $\left(\mathrm{CH}_{2}\right), 35.19(\mathrm{CH}), 37.85\left(\mathrm{CH}_{2}\right), 38.68\left(\mathrm{CH}_{2}\right), 39.74(\mathrm{CH}), 52.81(\mathrm{CH}), 82.25(\mathrm{CH}), 130.19(\mathrm{C})$, 139.91 (C), 177.11 (C); IR (ATR) $v=2959,2937,2910,2877,2856,2056,1843,1755,1684,1654$, 1635, 1486, 1457, 1436, 1378, 1343, 1311, 1292, 1236, 1199, 1169, 1051, 1037, 994, 940, 885, 868, 821, 795, 744, 722, 684, $621 \mathrm{~cm}^{-1}$; GC-MS (EI): m/z = 220 (73), 205 (77), 177 (23), 164 (14), 163 (14), 159 (11), 149 (13), 145 (22), 139 (36), 137 (22), 131 (16), 121 (19), 119 (27), 111 (27), 109 (100), 105 (39), 93 (31), 91 (72), 81 (85), 79 (90), 77 (64), 67 (36), 65 (26), 55 (53), 53 (40), 41 (72), 39 (49); HRMS (ESI-TOF): m/z calcd for $\mathrm{C}_{14} \mathrm{H}_{20} \mathrm{O}_{2} \mathrm{Na}$ [M+Na] $]^{+}: 243.1356$; found: 243.1355.

\section{(-)-Osmitopsin (1)}
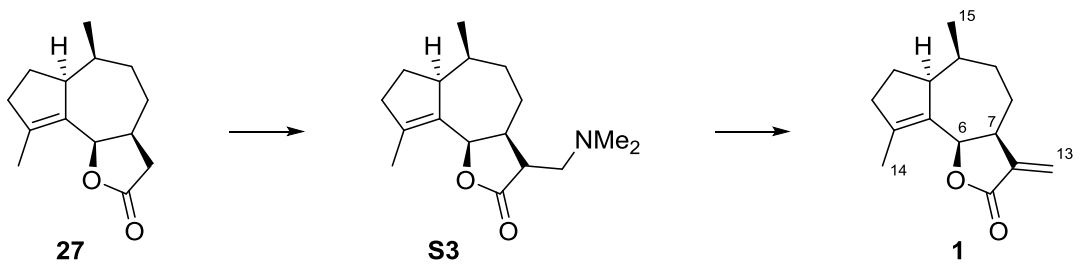

To a solution of hexamethyldisilazane $(475 \mu \mathrm{L}, 2.30 \mathrm{mmol})$ in $\mathrm{THF}(2.8 \mathrm{~mL})$ at $-78{ }^{\circ} \mathrm{C}$ was added BuLi (1.64 mL, $2.30 \mathrm{mmol}, 1.4 \mathrm{M}$ in hexane). The mixture was warmed to $0{ }^{\circ} \mathrm{C}$ for $1 \mathrm{~h}$ and then cooled to $-78{ }^{\circ} \mathrm{C}$ again. A solution of lactone 27 (44.8 mg, $\left.203 \mu \mathrm{mol}\right)$ in THF (4 mL) was added dropwise to the LiHMDS solution at $-78^{\circ} \mathrm{C}$. After stirring at this temperature for $1.5 \mathrm{~h}$, Eschenmoser salt 25 (639.4 mg, $3.46 \mathrm{mmol})$ was added in a countercurrent of argon. The mixture was stirred for 30 min at $-78{ }^{\circ} \mathrm{C}$, then it was warmed to $-30{ }^{\circ} \mathrm{C}$, and stirring was continued for additional $4 \mathrm{~h}$. After complete conversion was indicated by TLC, the reaction was quenched through addition of aqueous $\mathrm{HCl}$ (2 M, until slightly acidic), $\mathrm{K}_{2} \mathrm{CO}_{3}$ (until slightly basic), and water (5 mL). The aqueous layer was extracted with ethyl acetate, and the organic layer was dried over magnesium sulfate. The solvents were removed under reduced pressure, and the residue was dissolved in methanol $(7.8 \mathrm{~mL})$ and treated with methyl iodide $(240 \mu \mathrm{L}, 3.86 \mathrm{mmol})$. After stirring at room temperature overnight, methanol was removed under reduced pressure. The residue was dissolved in THF $(7.7 \mathrm{~mL})$ and treated with DBU 
(60 $\mu \mathrm{L}, 402 \mu \mathrm{mol})$. The mixture was stirred for $2 \mathrm{~h}$ at room temperature, then water $(10 \mathrm{~mL})$ was added, and the aqueous layer was extracted with ethyl acetate. After drying over magnesium sulfate, column chromatography (pentane/ethyl acetate, $20 / 1 \rightarrow 10 / 1)$ yielded osmitopsin $(1,15.9 \mathrm{mg}, 34 \%)$ as a slightly yellow oil that turned into a yellowish wax upon storage at $-32{ }^{\circ} \mathrm{C}^{3}$

$\mathrm{R}_{\mathrm{f}}=0.38$ (pentane/ethyl acetate, 10/1); $[\alpha]_{\mathrm{D}}{ }^{21}-4.1\left(c 0.85, \mathrm{CHCl}_{3}\right) ;{ }^{1} \mathrm{H}$ NMR $\left(600 \mathrm{MHz}, \mathrm{CCl}_{4}+0.5\right.$ vol\% tetramethylsilane, $\mathrm{C}_{6} \mathrm{D}_{6}$ capillary): $\delta 0.89(\mathrm{~d}, J=6.8 \mathrm{~Hz}, 3 \mathrm{H}), 1.40-1.48(\mathrm{~m}, 1 \mathrm{H}), 1.53-1.62(\mathrm{~m}$, $3 \mathrm{H}), 1.71-1.76(\mathrm{~m}, 1 \mathrm{H}), 1.77(\mathrm{~s}, 3 \mathrm{H}), 1.79-1.85(\mathrm{~m}, 1 \mathrm{H}), 1.90-1.96(\mathrm{~m}, 1 \mathrm{H}), 2.19-2.33(\mathrm{~m}, 2 \mathrm{H})$, 2.71-2.77 (m, 1H), 3.08-3.14 (m, 1H), 5.21 (d, $J=8.3 \mathrm{~Hz}, 1 \mathrm{H}), 5.43(\mathrm{~d}, J=2.7 \mathrm{~Hz}, 1 \mathrm{H}), 6.17$ (d, $J=$ $2.7 \mathrm{~Hz}, 1 \mathrm{H}) ;{ }^{13} \mathrm{C}$ NMR $\left(150 \mathrm{MHz}, \mathrm{CCl}_{4}+0.5\right.$ vol\% tetramethylsilane, $\mathrm{C}_{6} \mathrm{D}_{6}$ capillary): $\delta 14.79\left(\mathrm{CH}_{3}\right)$, $18.06\left(\mathrm{CH}_{3}\right), 24.86\left(\mathrm{CH}_{2}\right), 28.68\left(\mathrm{CH}_{2}\right), 30.96\left(\mathrm{CH}_{2}\right), 32.65(\mathrm{CH}), 38.61\left(\mathrm{CH}_{2}\right), 41.65(\mathrm{CH}), 52.28$ $(\mathrm{CH}), 79.18(\mathrm{CH}), 120.63\left(\mathrm{CH}_{2}\right), 131.08(\mathrm{C}), 136.12$ (C), 139.96 (C), $167.49(\mathrm{C})$ : IR (ATR): v = 2949, 2924, 2858, 2138, 2056, 2030, 2010, 1942, 1918, 1868, 1844, 1820, 1760, 1717, 1699, 1684, 1653, 1635, 1452, 1376, 1320, 1265, 1192, 1128, 1103, 1028, 937, 813, 730, 685, $622 \mathrm{~cm}^{-1}$; GC-MS (EI): m/z = 232 (46), 217 (16), 123 (81), 121 (12), 109 (100), 107 (17), 104 (14), 95 (21), 93 (20), 91 (28), 81 (33), 79 (50), 77 (26), 67 (14), 65 (11), 55 (16), 53 (31), 41 (22), 39 (15); HRMS (ESI-TOF): m/z calcd for $\mathrm{C}_{15} \mathrm{H}_{20} \mathrm{O}_{2} \mathrm{Na}[\mathrm{M}+\mathrm{Na}]^{+}$: 255.1356; found: 255.1353 .

Table 3: Chemical shifts $[\mathrm{ppm}]$ of synthetic $\mathbf{1}\left(600 \mathrm{MHz}, \mathrm{CCl}_{4}+\right.$ tetramethylsilane $)$ and natural $\mathbf{1}^{3}$ $\left(100 \mathrm{MHz}, \mathrm{CCl}_{4}+\right.$ tetramethylsilane).

\begin{tabular}{lll}
\hline & \multicolumn{1}{c}{ Synthetic osmitopsin (1) } & \multicolumn{1}{c}{ Natural osmitopsin (1) } \\
\hline $15-\mathrm{H}$ & $0.89(\mathrm{~d}, J=6.8 \mathrm{~Hz})$ & $0.91(\mathrm{~d}, J=6.5 \mathrm{~Hz})$ \\
$14-\mathrm{H}$ & $1.77(\mathrm{~s})$ & $1.79(\mathrm{br} \mathrm{s})$ \\
$13-\mathrm{H}$ & $5.43(\mathrm{~d}, J=2.7 \mathrm{~Hz})$ & $5.44(\mathrm{dd}, J=2.4 \mathrm{~Hz}, 0.5 \mathrm{~Hz})$ \\
$13-\mathrm{H}^{\prime}$ & $6.17(\mathrm{~d}, J=2.7 \mathrm{~Hz})$ & $6.16(\mathrm{dd}, J=2.8 \mathrm{~Hz}, 0.5 \mathrm{~Hz})$ \\
$7-\mathrm{H}$ & $3.11(\mathrm{~m})$ & $3.14(\mathrm{~m})$ \\
$6-\mathrm{H}$ & $5.21(\mathrm{~d}, J=8.3 \mathrm{~Hz})$ & $5.25(\mathrm{br} \mathrm{d}, J=7.0 \mathrm{~Hz})$ \\
\hline
\end{tabular}


Epoxidation of (-)-osmitopsin (1) to give guaianolide $\mathbf{3}$
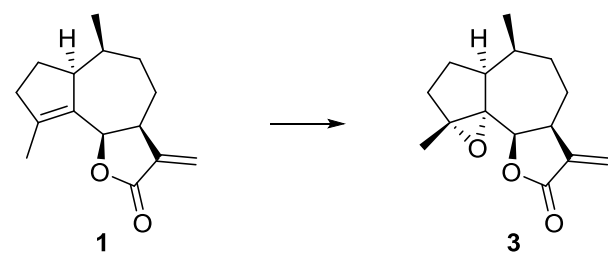

(-)-Osmitopsin $(\mathbf{1}, 16.9 \mathrm{mg}, 72.7 \mu \mathrm{mol})$ was dissolved in dry chloroform $(1.7 \mathrm{~mL})$. Solid $m \mathrm{CPBA}$ (55.4 mg, $247 \mu \mathrm{mol}$ ) was added, and the mixture was stirred for $2 \mathrm{~h}$ at ambient temperature. After addition of saturated aqueous $\mathrm{Na}_{2} \mathrm{SO}_{3}(1 \mathrm{~mL})$, the aqueous layer was extracted with ethyl acetate. The combined organic layers were washed with saturated aqueous $\mathrm{NaHCO}_{3}$ to remove the resultant $\mathrm{m}$ chlorobenzoic acid. Flash chromatography (pentane/ethyl acetate, 2/1) gave $\alpha$-methylene lactone 3 (16.6 mg, 92\%) as a colorless oil. ${ }^{3} \mathrm{R}_{\mathrm{f}}=0.46$ (pentane/ethyl acetate, 2/1). For further data, see preparation of 3 by DIBAL reduction of enamino lactone 24 .

(1) Li, T.-S.; Li, J.-T.; Li, H.-Z. J. Chromatogr. A 1995, 715, 372-375.

(2) Knüppel, S.; Rogachev, V. O.; Metz, P. Eur. J. Org. Chem. 2010, 6145-6148.

(3) Bohlmann, F.; Zdero, C. Chem. Ber. 1974, 107, 1409-1415. 


\section{Spectra}
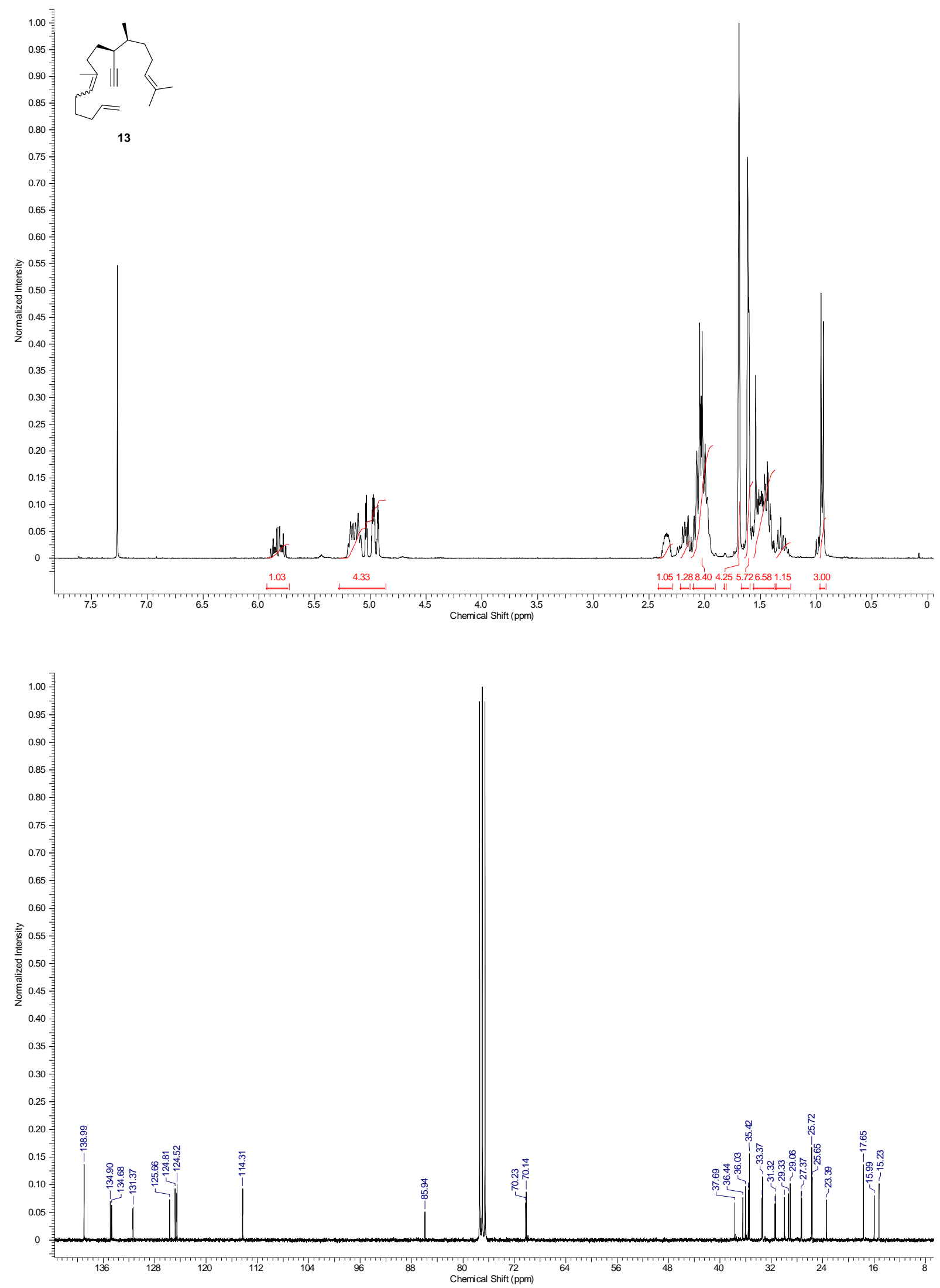

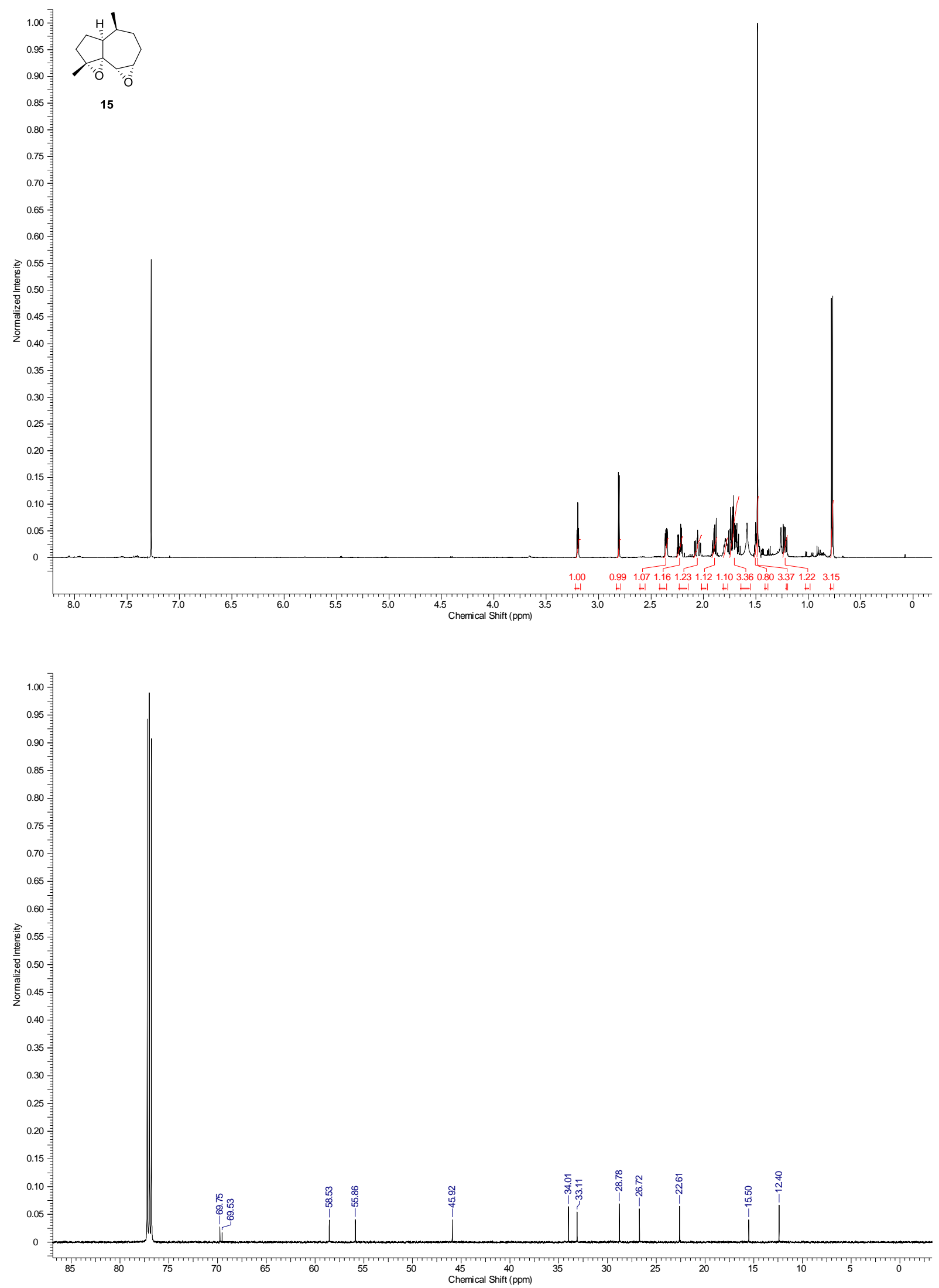

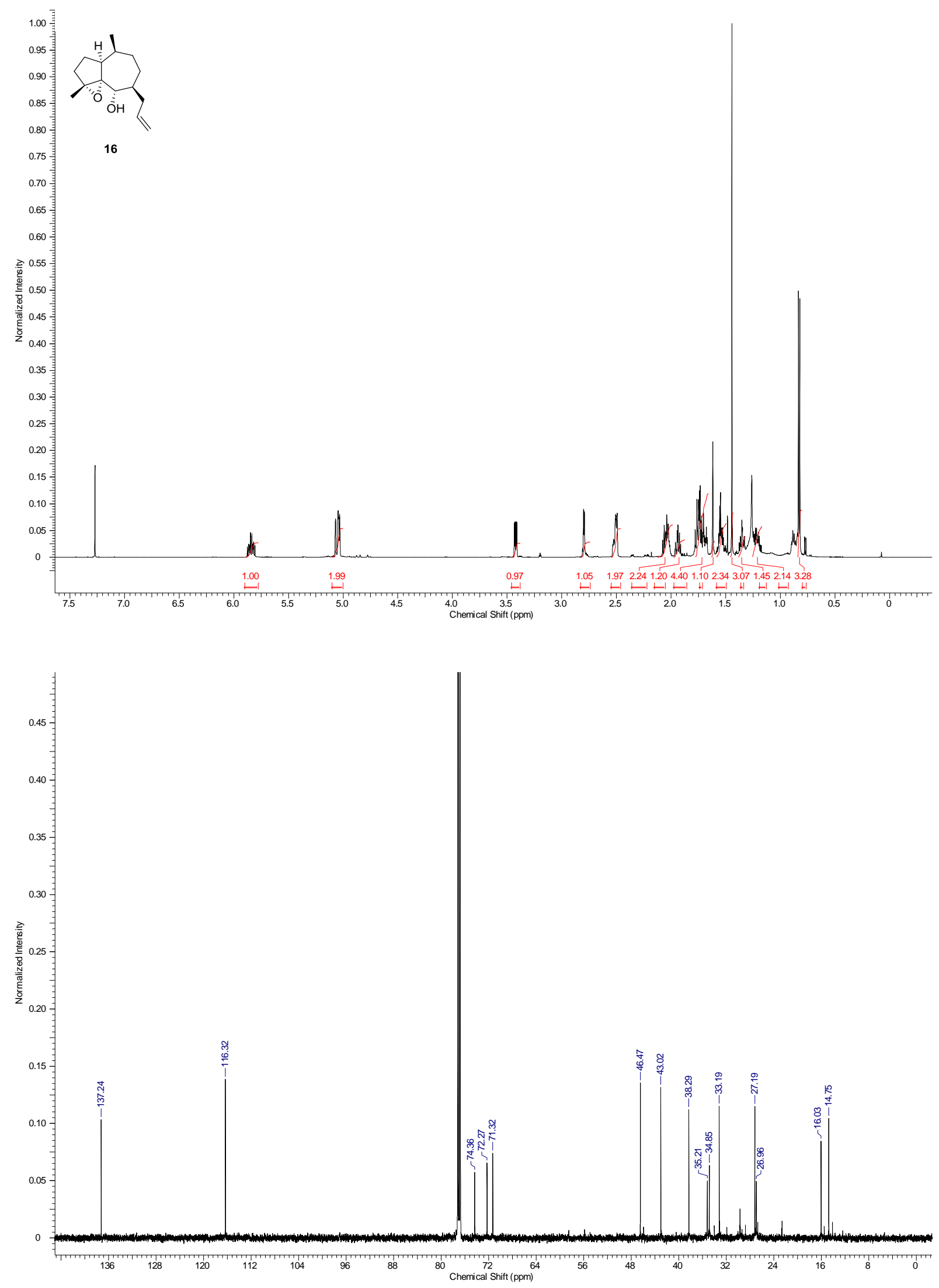

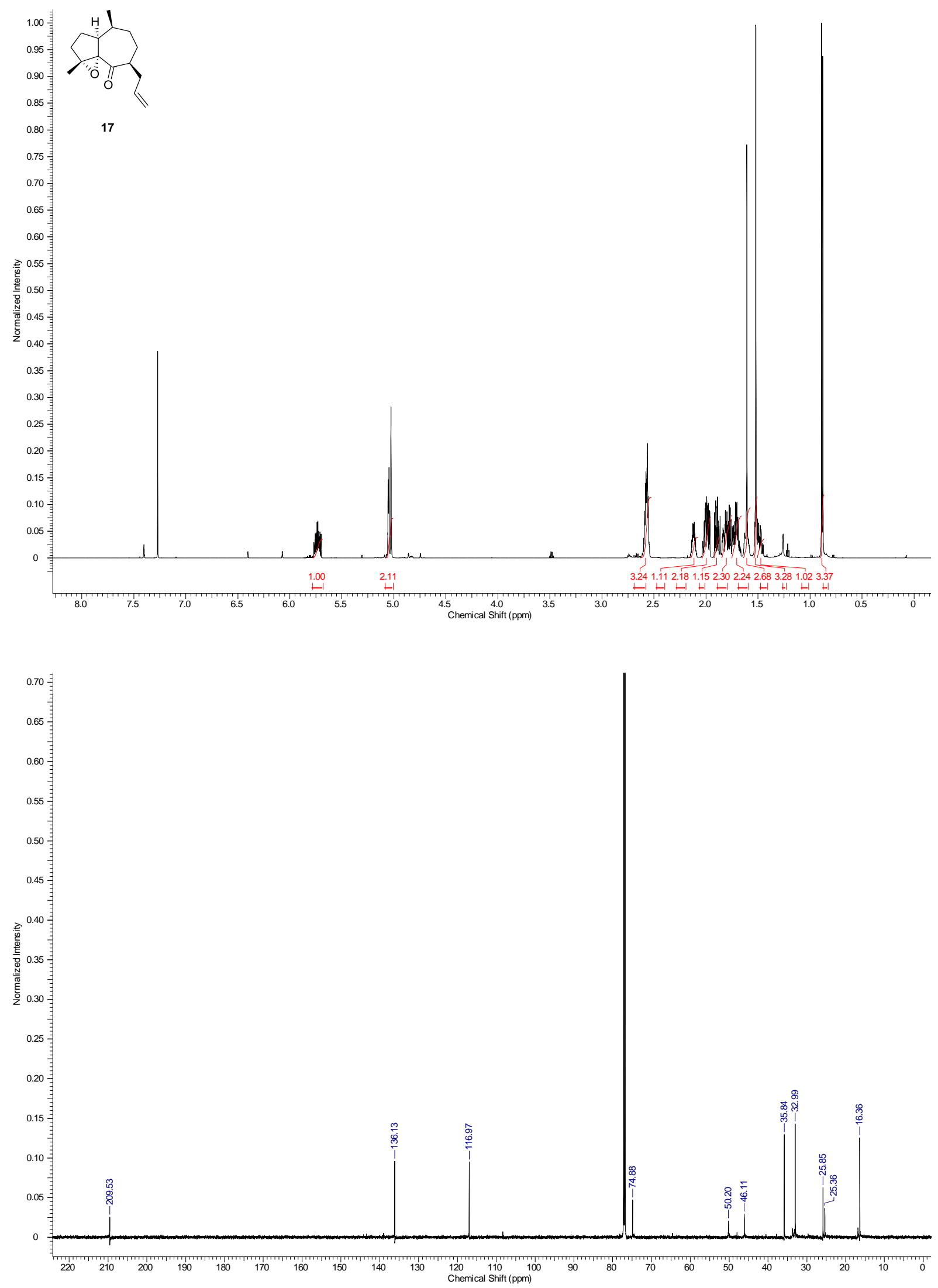

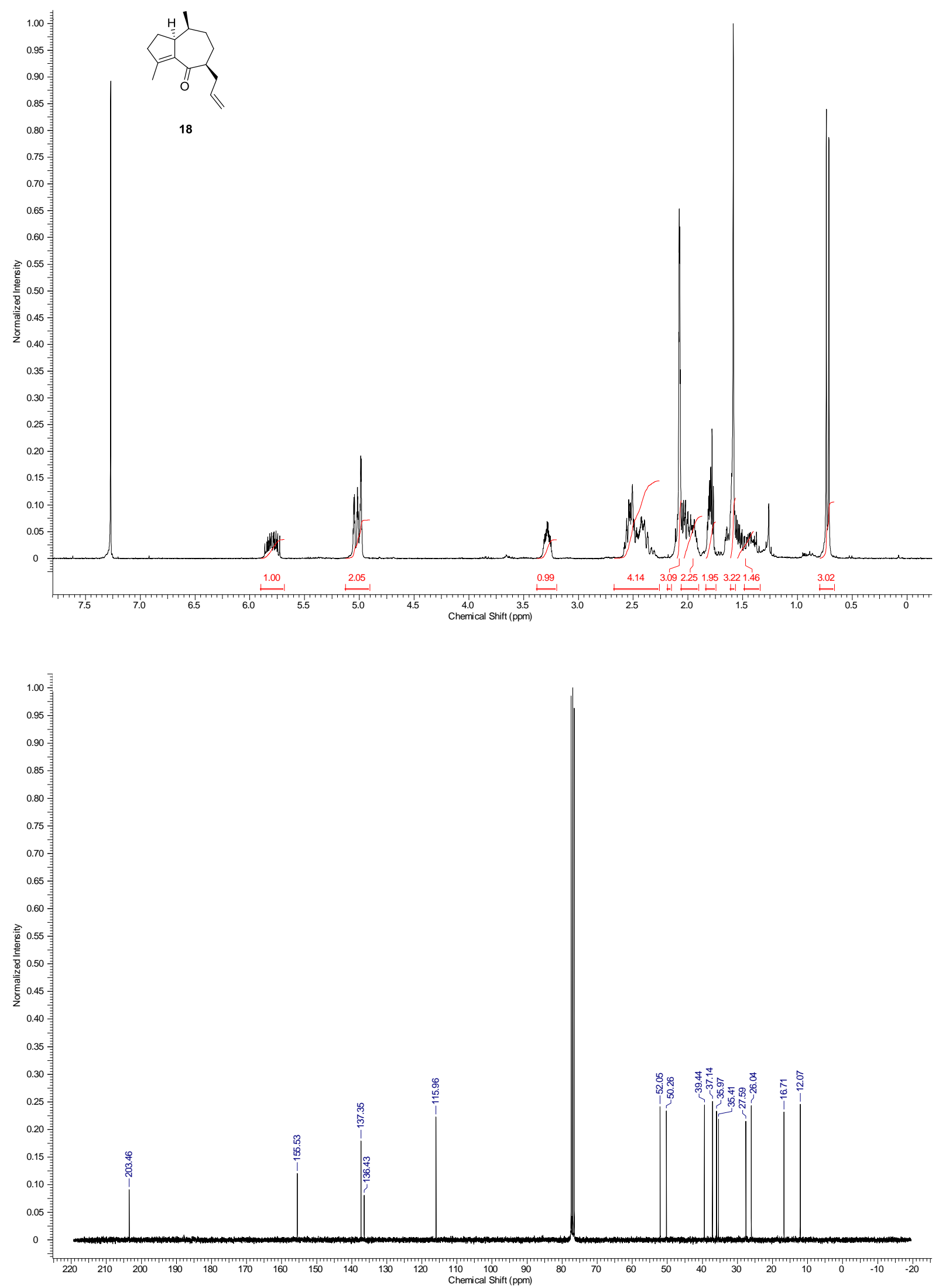

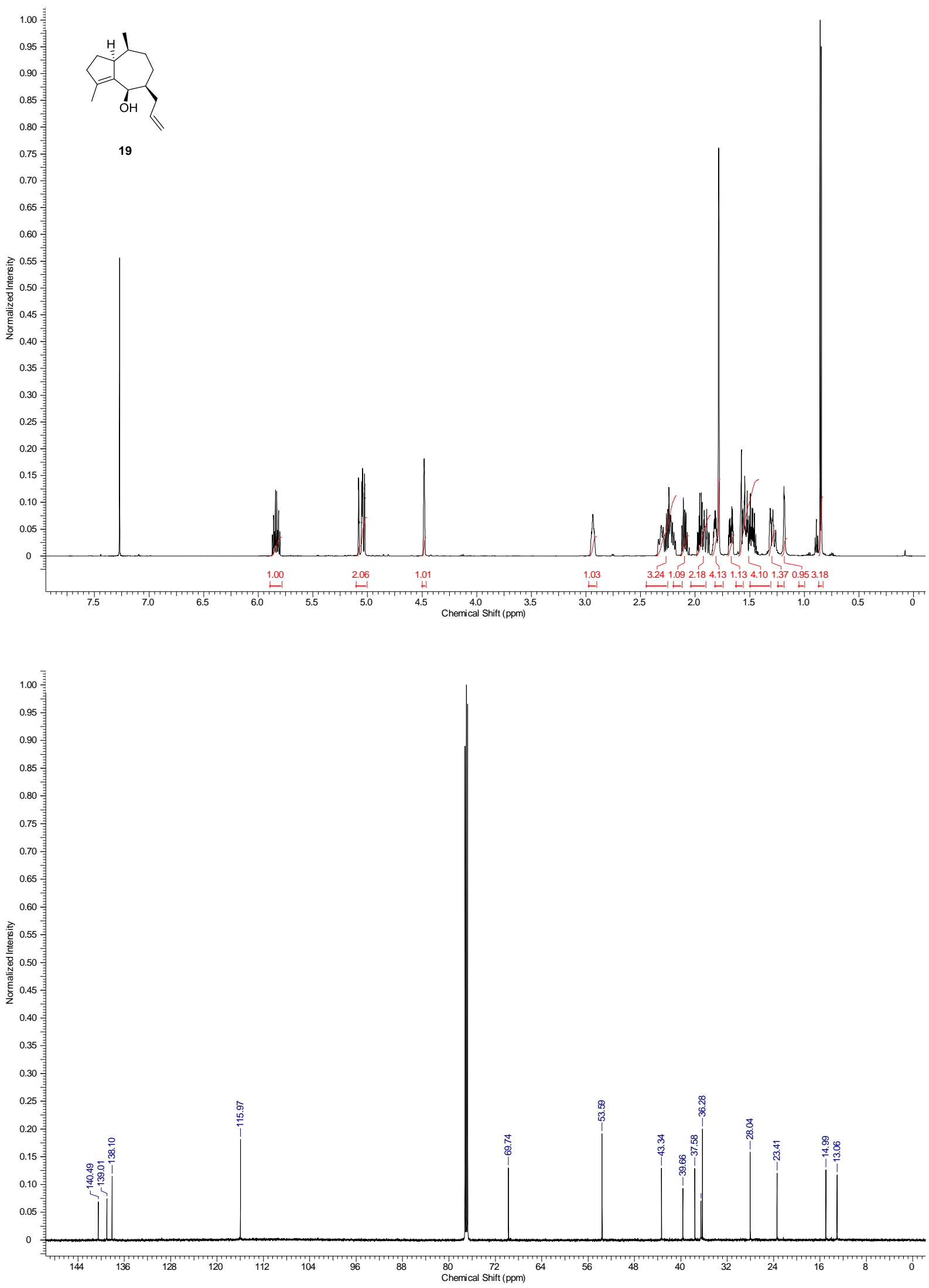

S26 

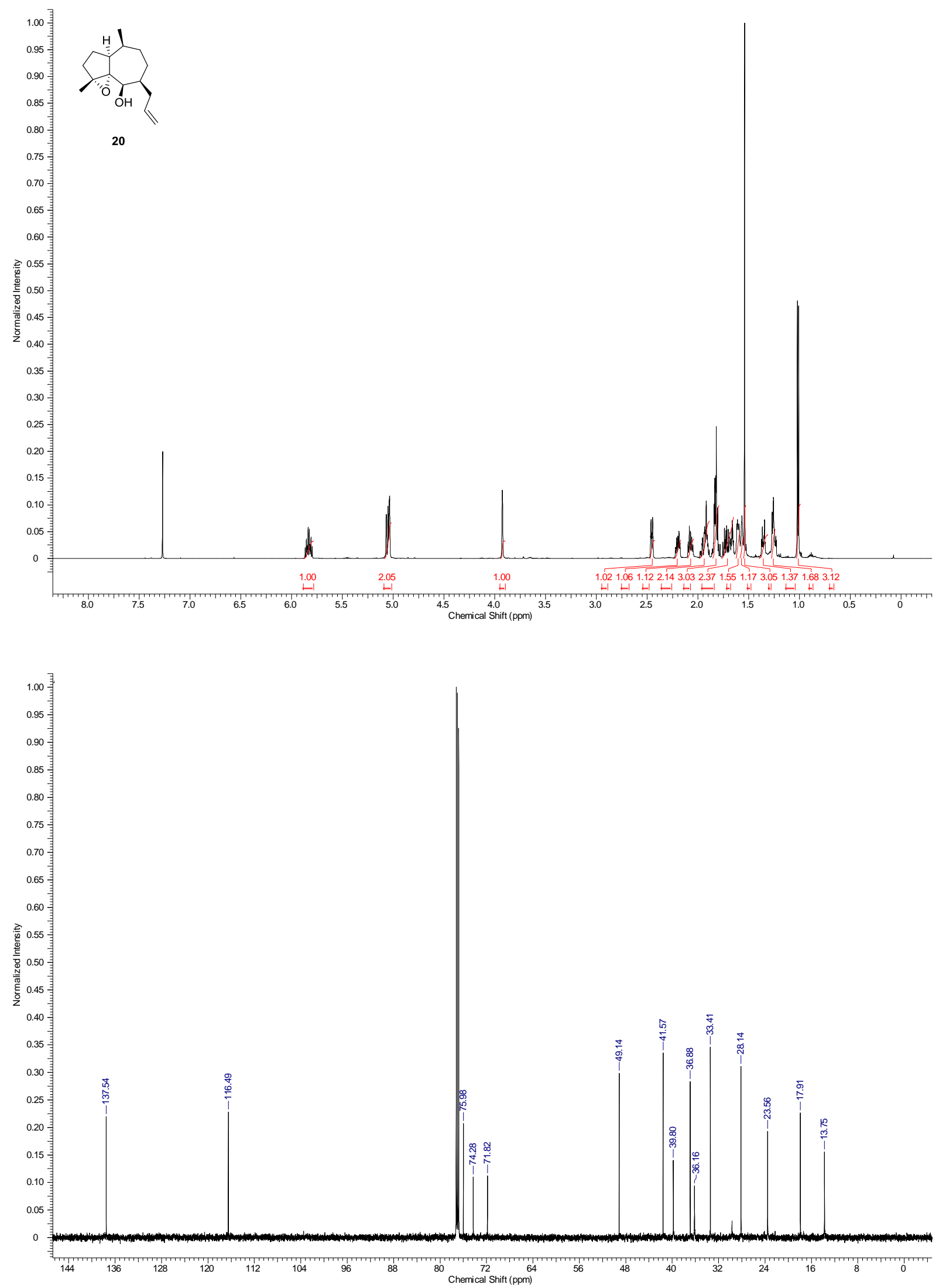

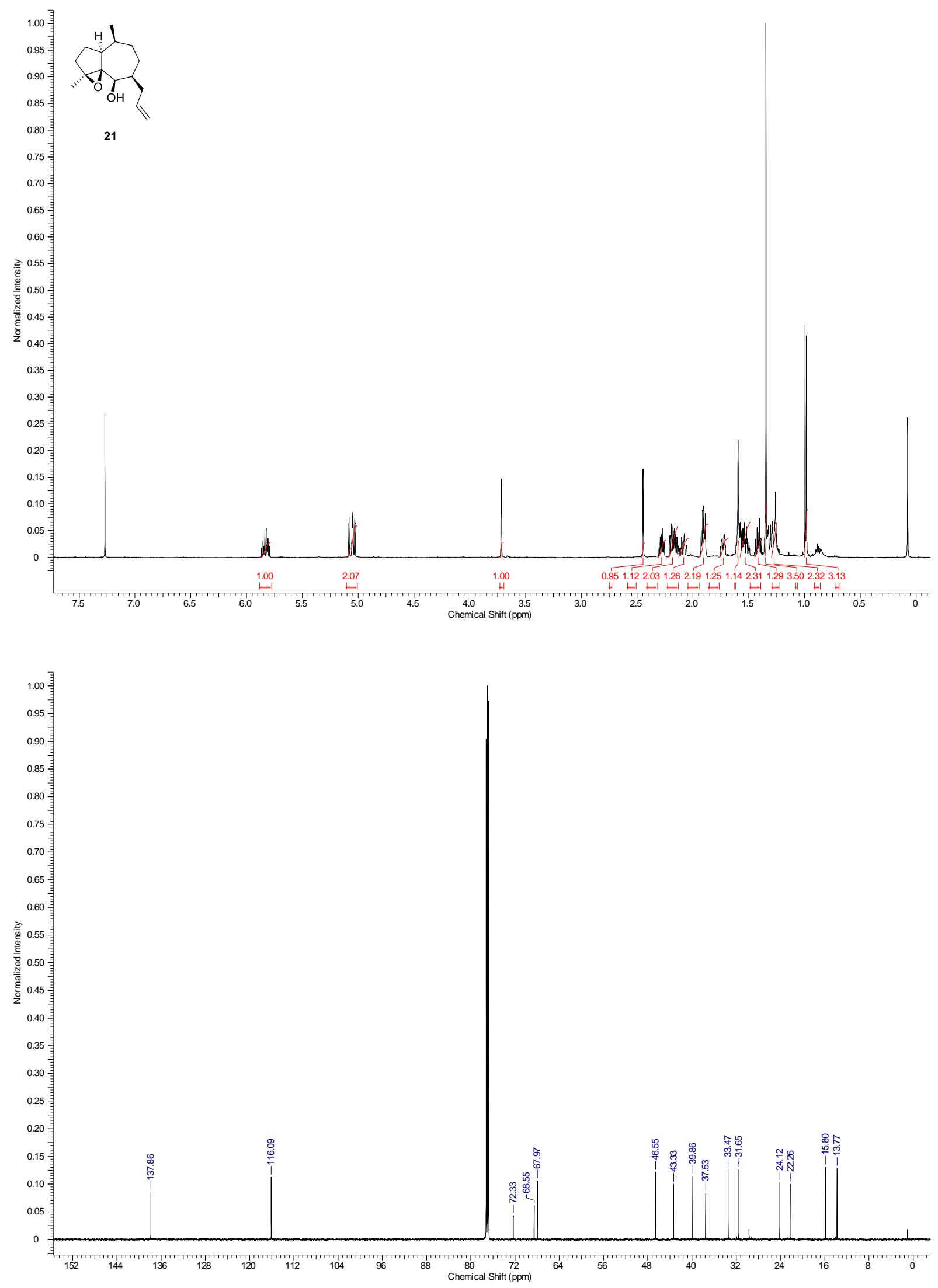

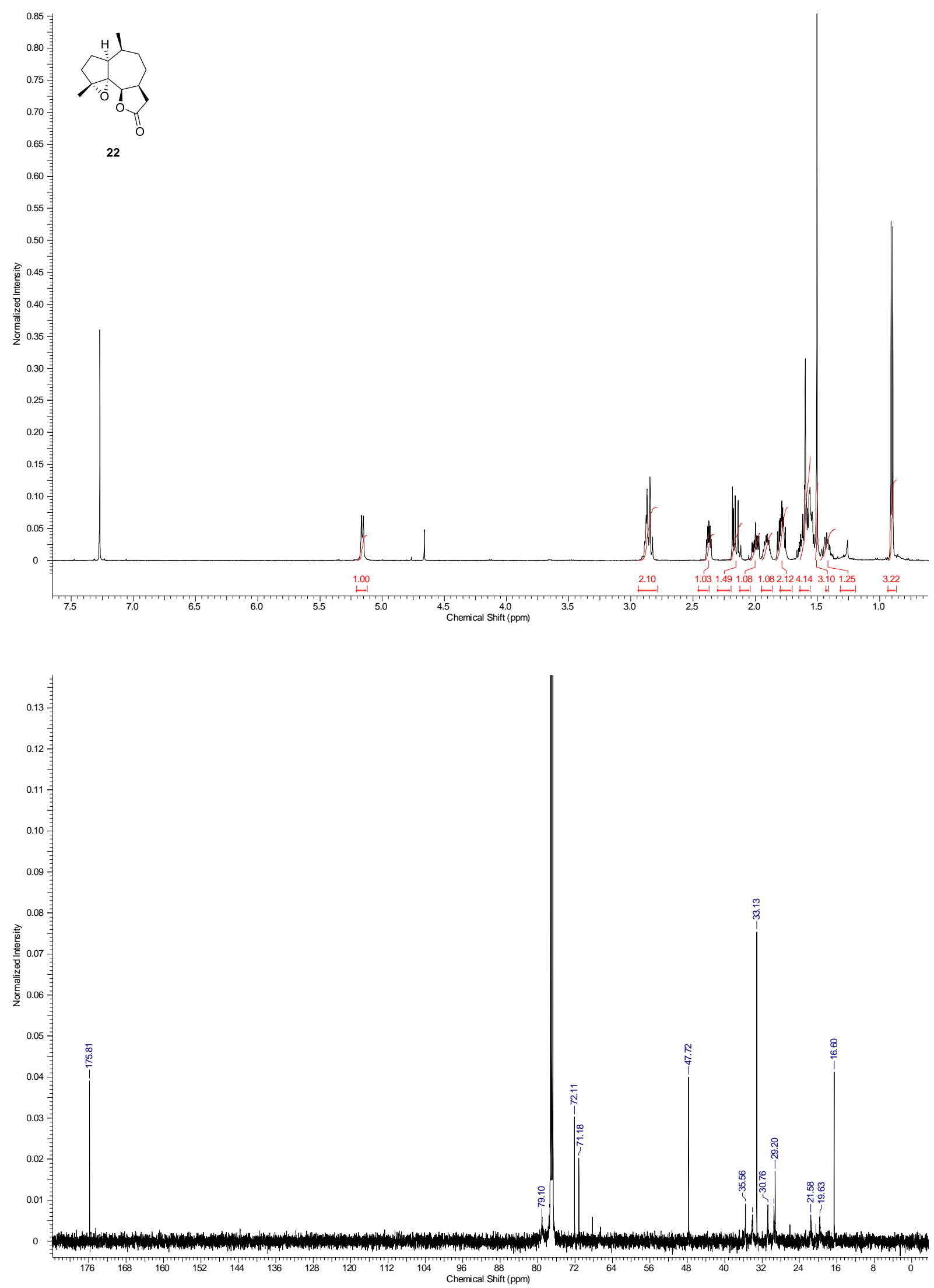

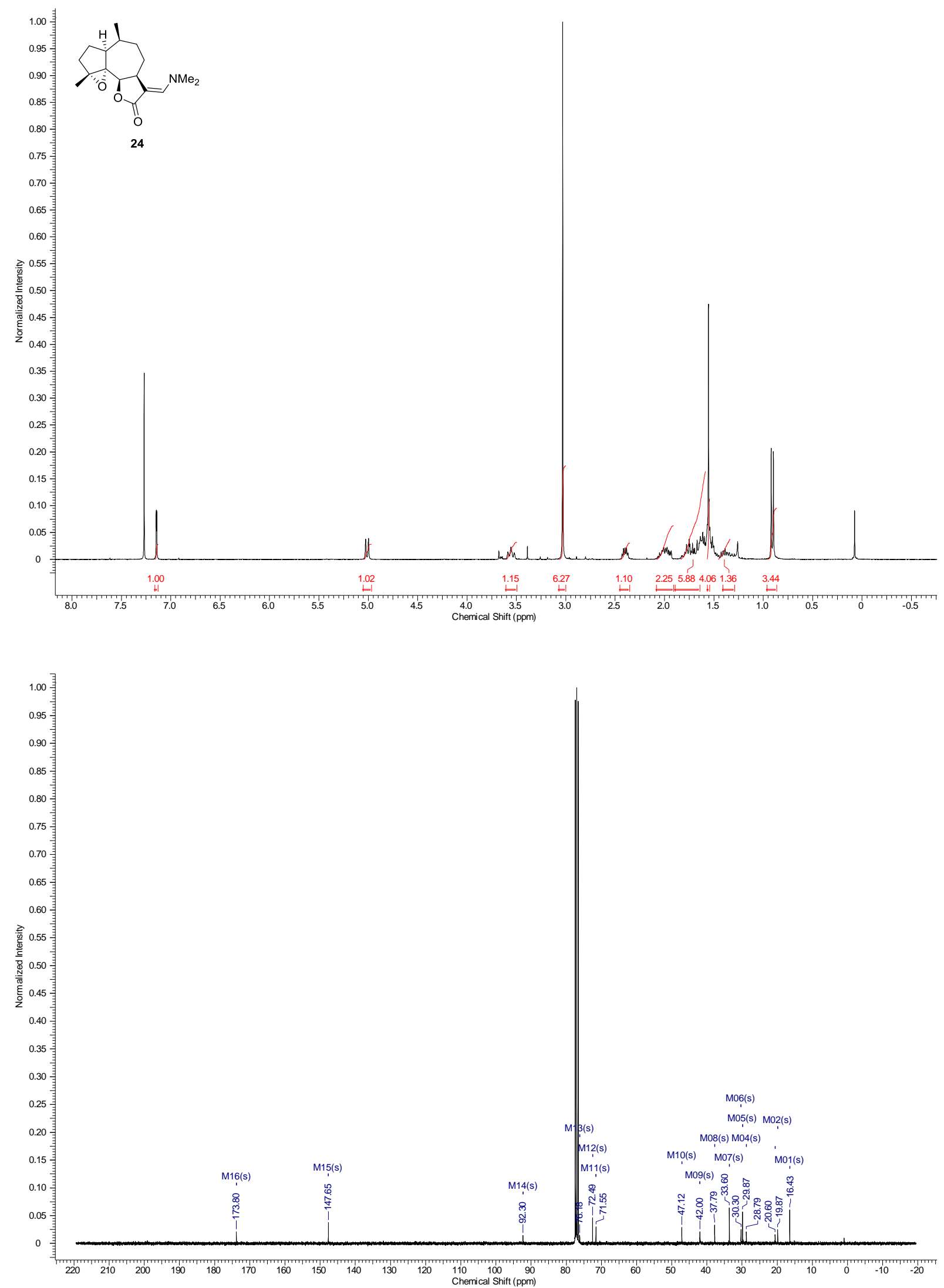

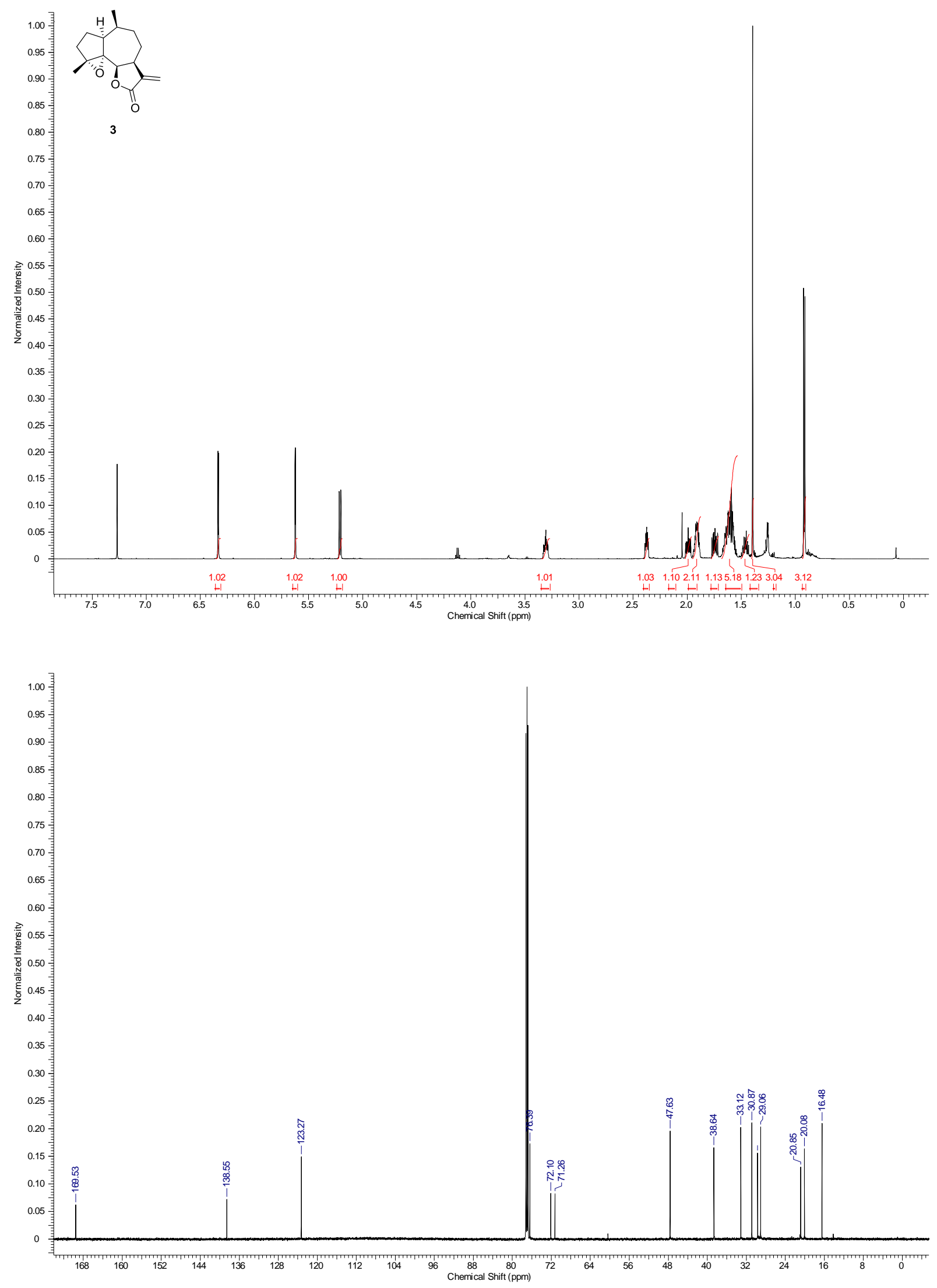

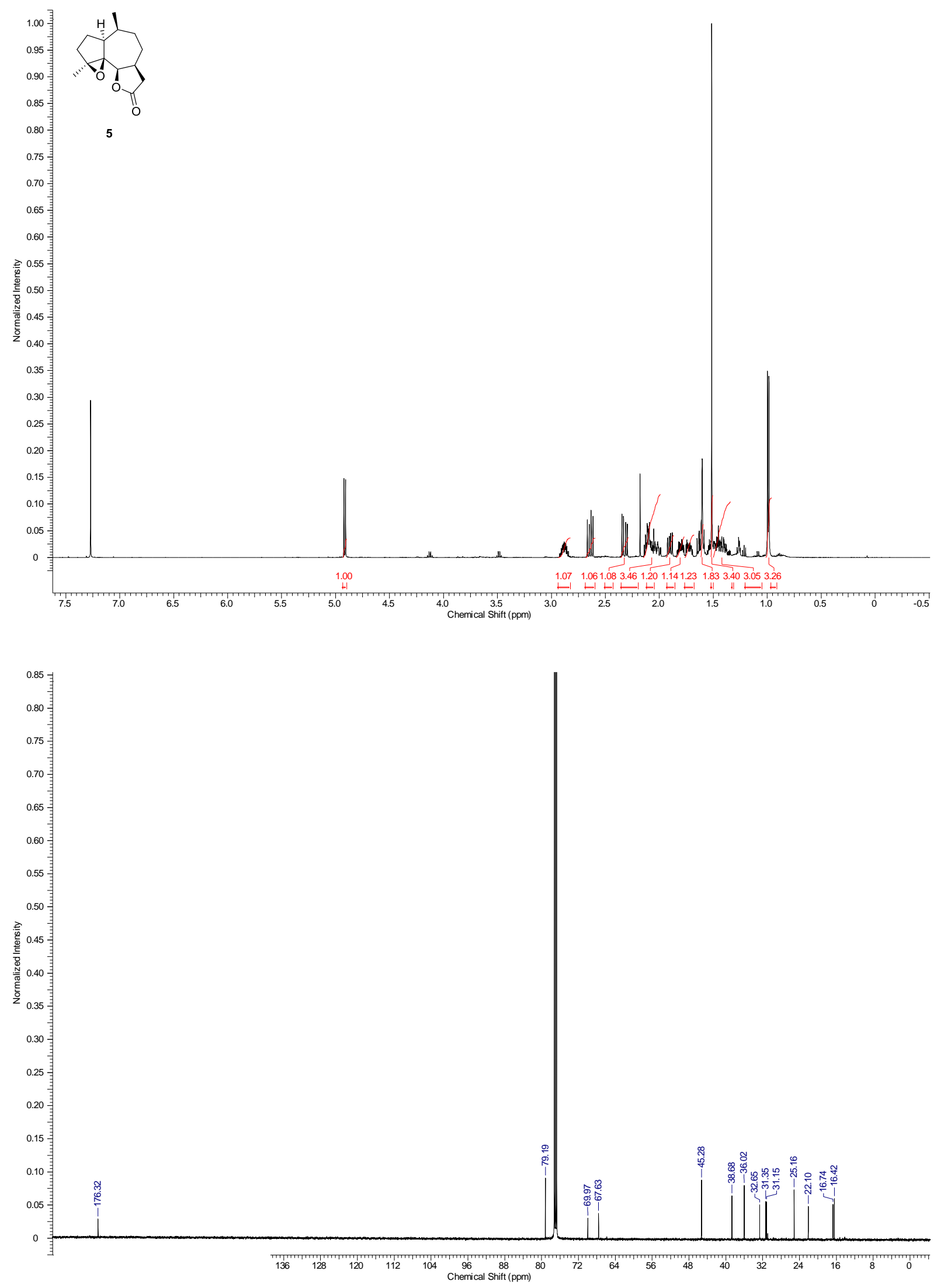

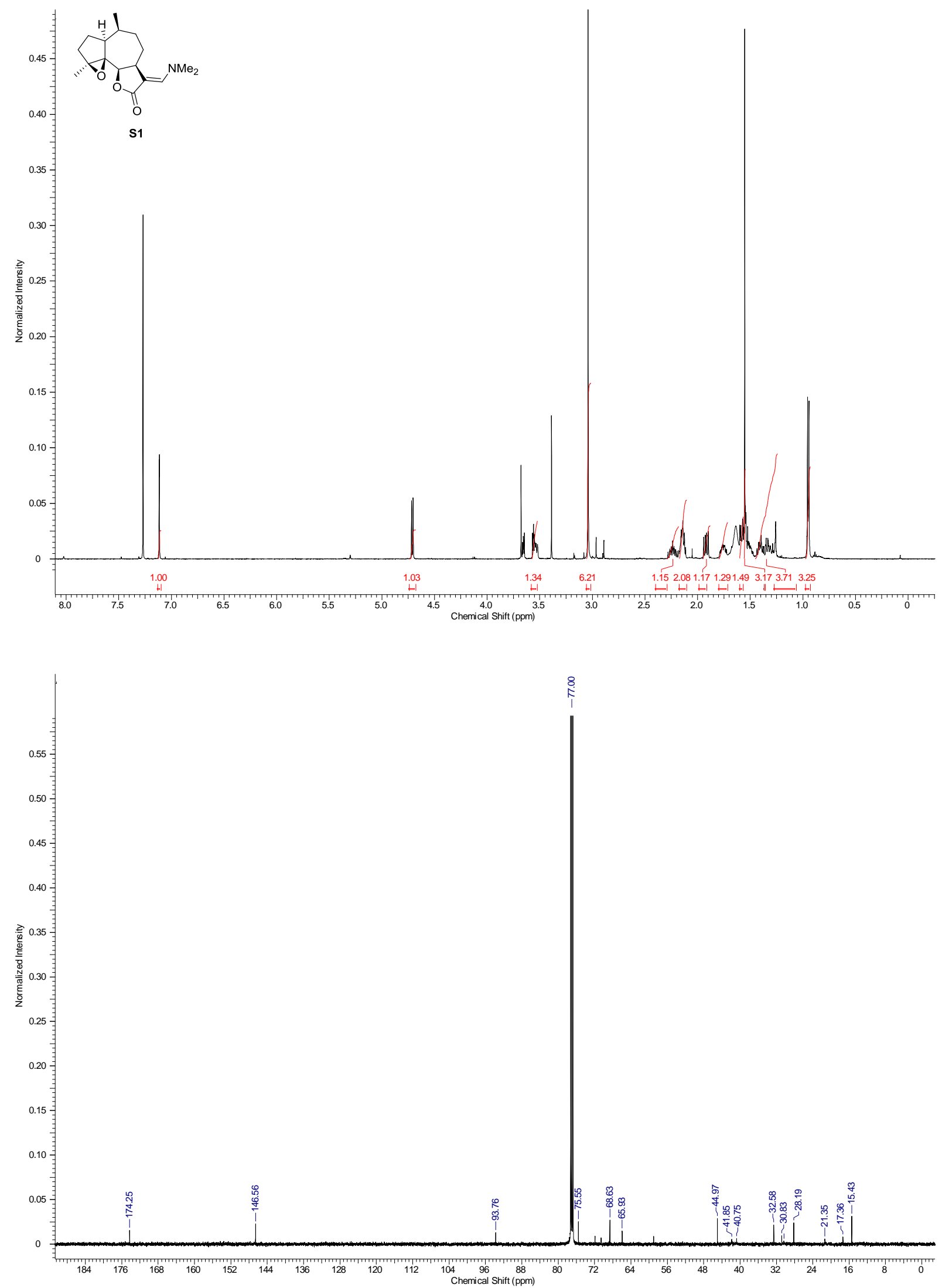

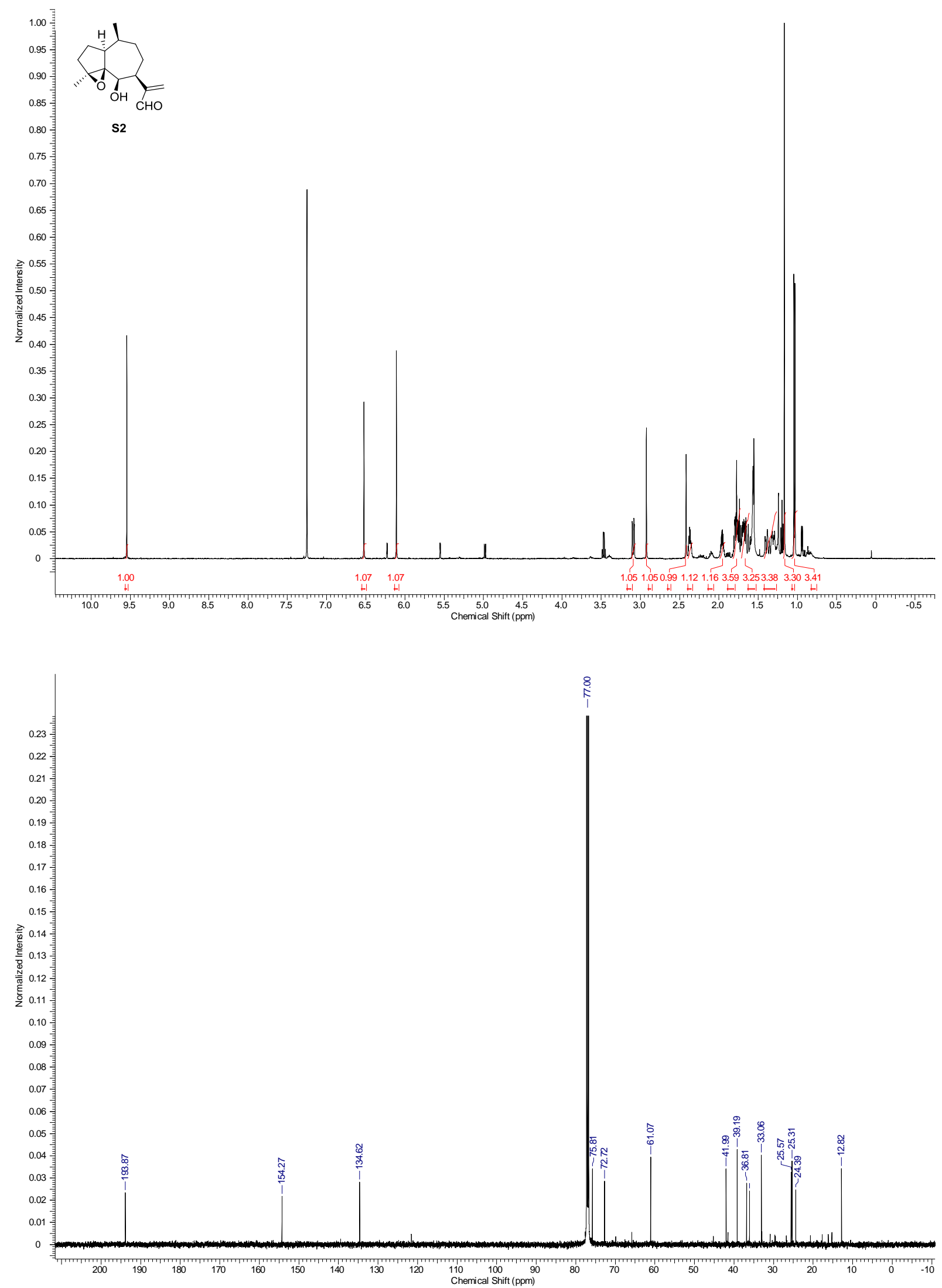

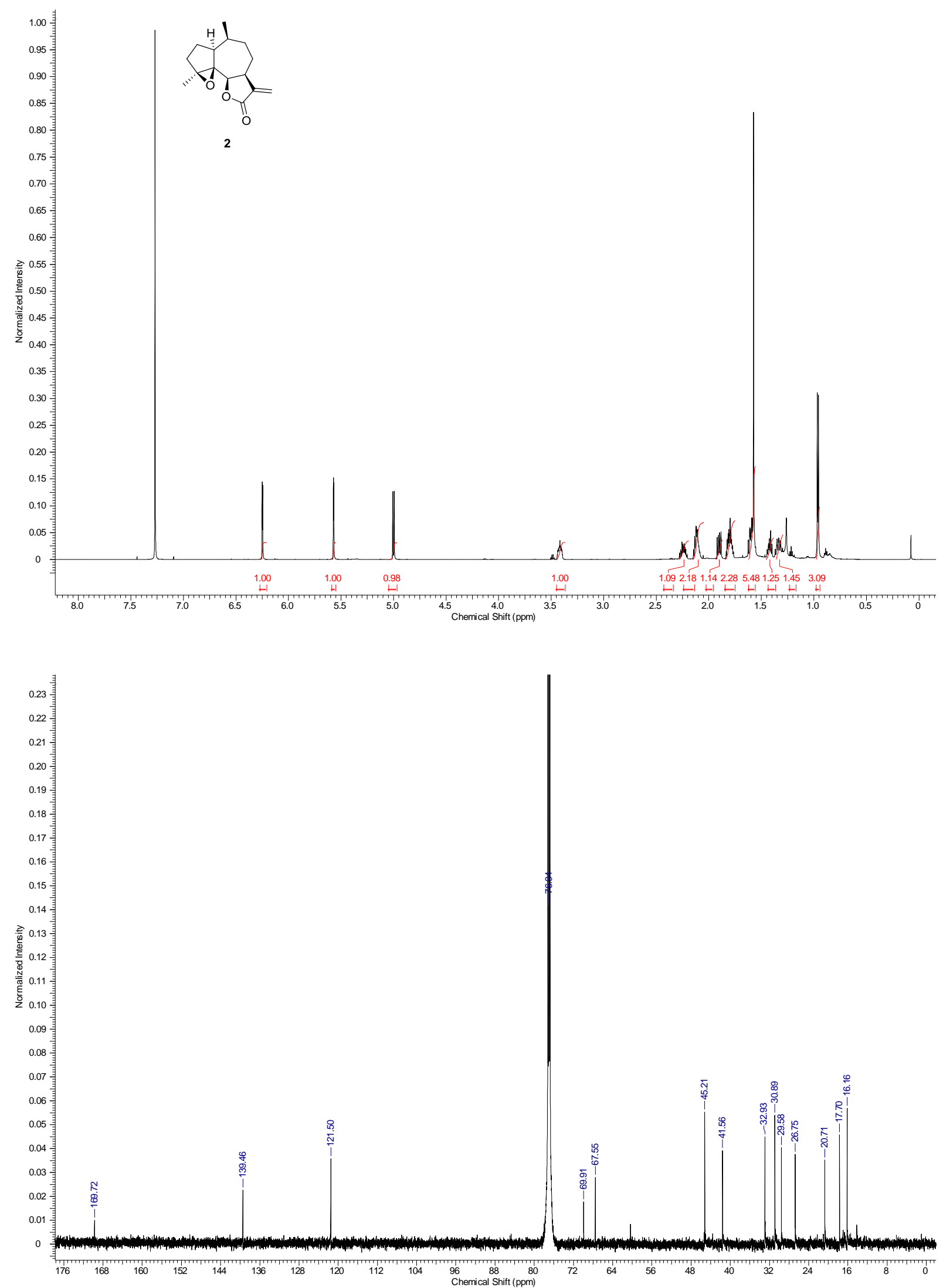

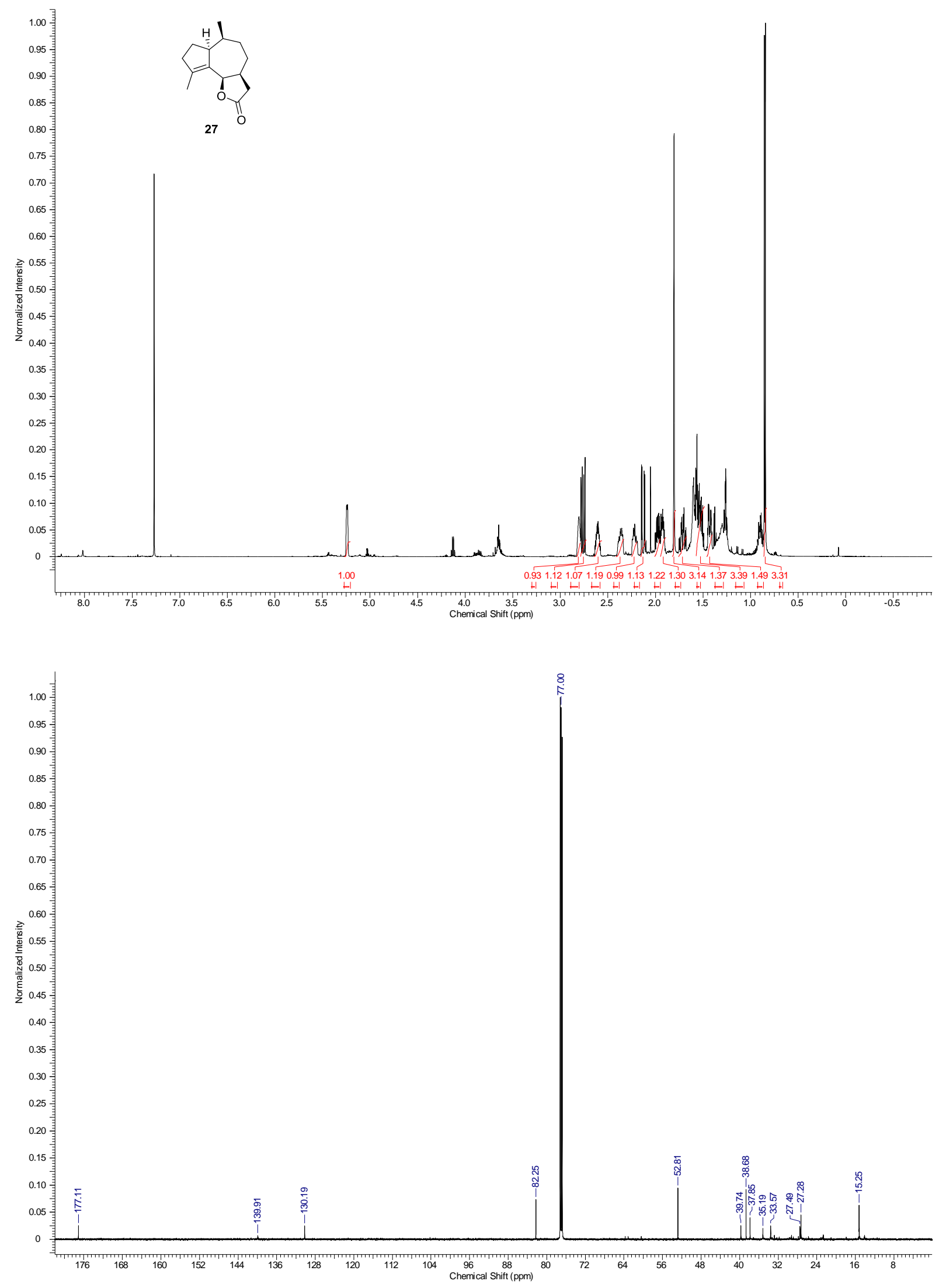

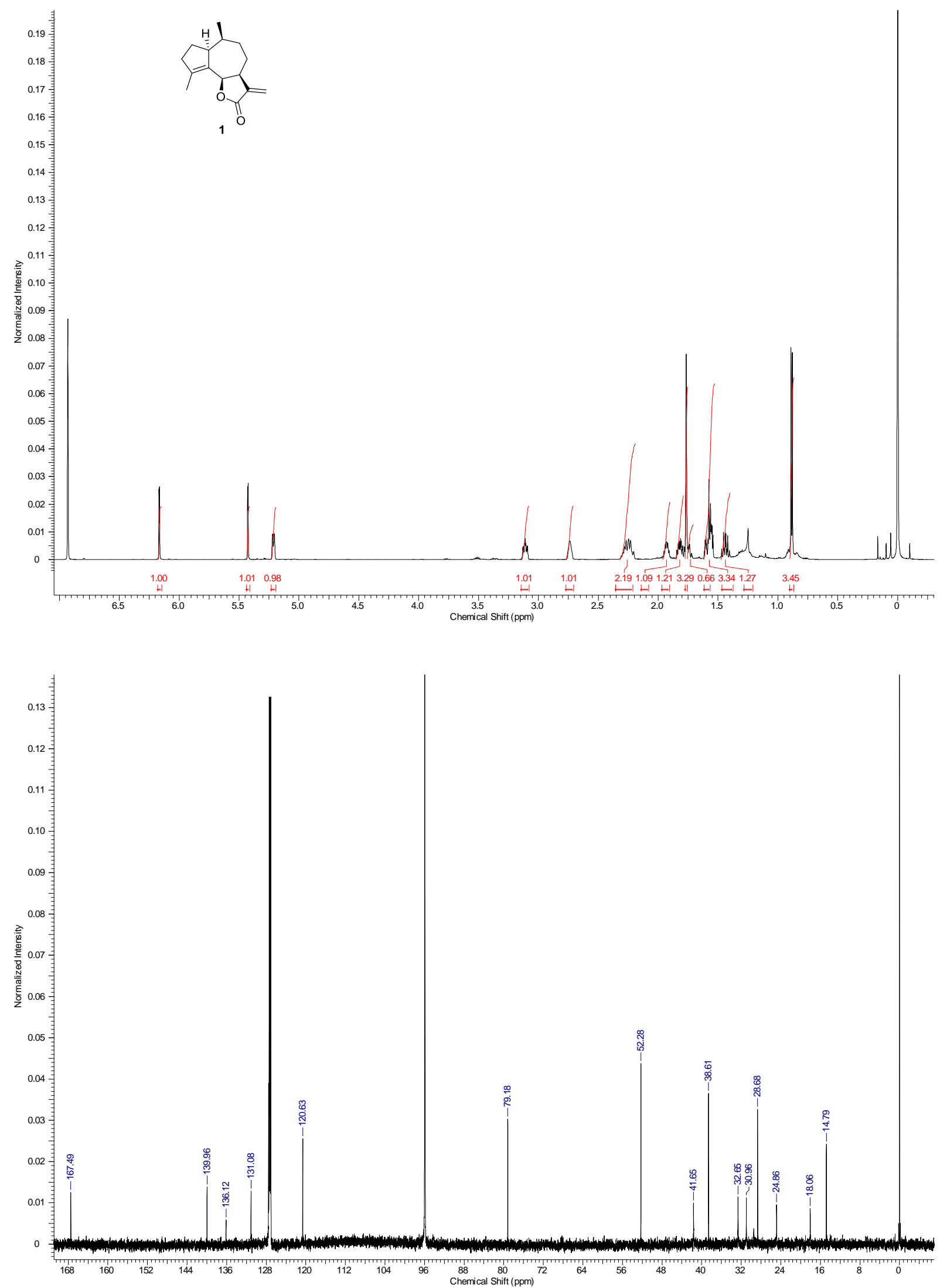